\title{
Illustrations and supporting texts for sound standing waves of air columns in pipes in introductory physics textbooks
}

\author{
Liang Zeng, ${ }^{1, *}$ Chris Smith, ${ }^{2}$ G. Herold Poelzer, ${ }^{3}$ Jennifer Rodriguez, ${ }^{1}$ \\ Edgar Corpuz, ${ }^{1}$ and George Yanev ${ }^{4}$ \\ ${ }^{1}$ Department of Physics and Geology, The University of Texas-Pan American, \\ Edinburg, Texas 78539, USA \\ ${ }^{2}$ Department of Chemistry, The University of Texas-Pan American, Edinburg, Texas 78539, USA \\ ${ }^{3}$ Department of Educational Psychology, The University of Texas-Pan American, \\ Edinburg, Texas 78539, USA \\ ${ }^{4}$ Department of Mathematics, The University of Texas-Pan American, Edinburg, Texas 78539, USA
}

(Received 28 June 2013; published 30 July 2014)

\begin{abstract}
In our pilot studies, we found that many introductory physics textbook illustrations with supporting text for sound standing waves of air columns in open-open, open-closed, and closed-closed pipes inhibit student understanding of sound standing wave phenomena due to student misunderstanding of how air molecules move within these pipes. Based on the construct of meaningful learning from cognitive psychology and semiotics, a quasiexperimental study was conducted to investigate the comparative effectiveness of two alternative approaches to student understanding: a traditional textbook illustration approach versus a newly designed air molecule motion illustration approach. Thirty volunteer students from introductory physics classes were randomly assigned to two groups of 15 each. Both groups were administered a presurvey. Then, group A read the air molecule motion illustration handout, and group B read a traditional textbook illustration handout; both groups were administered postsurveys. Subsequently, the procedure was reversed: group B read the air molecule motion illustration handout and group A read the traditional textbook illustration handout. This was followed by a second postsurvey along with an exit research questionnaire. The study found that the majority of students experienced meaningful learning and stated that they understood sound standing wave phenomena significantly better using the air molecule motion illustration approach. This finding provides a method for physics education researchers to design illustrations for abstract sound standing wave concepts, for publishers to improve their illustrations with supporting text, and for instructors to facilitate deeper learning in their students on sound standing waves.
\end{abstract}

\section{INTRODUCTION}

Visuals play an important role in learning. This is reflected in our educational curricula which, more and more, contain visual representations such as graphs, pictures, and the like, that are designed to enhance student learning. Notwithstanding this effort at visual representations, the research literature reports that students, in general, still have great difficulty with interpreting graphs and diagrams in science [1-3]. And, with a more complex phenomenon such as sound waves, students ranging from nonscience majors taking conceptual physics courses to graduate students in physics misinterpret either graphical or pictorial representations of sound waves depicted as sinusoidal curves [4,5]. It comes as no surprise, then, that

\footnotetext{
*Corresponding author. zengl@utpa.edu

Published by the American Physical Society under the terms of the Creative Commons Attribution 3.0 License. Further distribution of this work must maintain attribution to the author $(s)$ and the published article's title, journal citation, and DOI.
}

physics education researchers find student explanations of sound wave phenomena to be mostly incorrect $[6,7]$. Why should this be the case?

We investigated the illustrations of sound standing waves in an air column in a pipe in thirteen algebra-based and calculus-based introductory physics textbooks [8-20] and a teacher's guide manual [21]. All illustrations were drawn with two intertwining sinusoidal curves, very much like those used for transverse standing waves. Three typical illustrations are shown in Fig. 1.

The amplitude of the sinusoidal waves is drawn beyond the walls of the tube in Fig. 1(a), touching the walls of the tube in Fig. 1(b), or inside the pipe with a small gap between the amplitude and the pipe walls in Fig. 1(c).

Besides the inconsistency of the drawn sinusoidal waves, there is no accompanying detailed text explaining what the two intertwining curves represent in the illustrations. Hence, we surmise that students would have difficulty interpreting sound standing waves with such illustrations.

Our study investigates the difficulties students have with interpreting the textbook illustrations of sound standing 


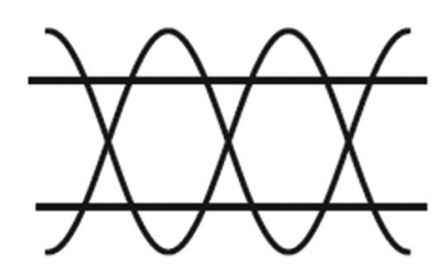

(a)

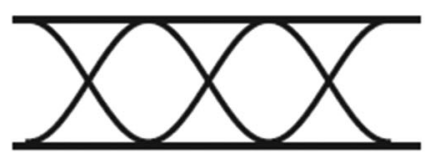

(b)

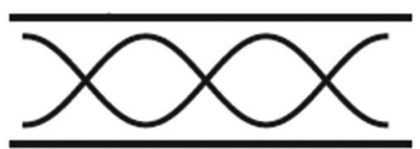

(c)

FIG. 1. Typical illustrations of sound standing waves in air columns in a pipe in introductory physics textbooks.

waves and, in light of the findings, proposes solutions to this problem.

\section{LITERATURE REVIEW}

In the literature review search, we found no studies on student conceptual understanding of sound standing waves. Therefore, in this literature review, we (i) look at student difficulties with interpreting pictorial representations of sound waves and (ii) use a pilot study to identify some of the problems with textbook representations of sound standing waves.

With regard to student reasoning about pictorial sound representations, students in a calculus-based introductory physics class at a large university chose one of three representations based on their reasoning about sound [5]. For example, those who chose the sine wave representation reasoned that the dust particle in the sound wave path moves up and down, and those who chose a roughly $70^{\circ}$ section of concentric circles representation reasoned that the dust particle moves along the sound propagation direction. In addition, those who chose the compression and rarefaction representation reasoned that the dust particle moves back and forth parallel to the direction of sound wave propagation.

In terms of student reasoning about sound, engineering students in an introductory physics course at the University of Maryland incorrectly reasoned that sound waves collide with the medium, either pushing air particles forward in a linear fashion or pushing air molecules forward along a sinusoidal path. Interviews indicated that these students perceived sound waves as objects [6].

Nonscience major students in a conceptual-physics class at Kansas State University also had incorrect reasoning of sound propagation: Some reasoned that sound propagates like an object moving through gaps in a medium. Others mixed wave properties of sound with their object perception [7]. These reasoning statements revealed that students are confused about sound wave travel.

To further investigate student reasoning about sound wave phenomena, we conducted a pilot study with a sample of 87 students from two different courses: (i) Physics for Scientists and Engineers and (ii) General Physics $[22,23]$. This study used five different textbook excerpts [8-12] to assess the comparative effectiveness of textbook illustrations on student conceptual understanding of sound standing waves pertaining to air molecule motion, the relationship between the amplitude of sound standing waves and the diameter of the pipe, and the difference between the pressure node and displacement node or between pressure antinodes and displacement antinodes.

If the illustrations in the textbooks shown to the students do not have accompanying explanative texts, students showed great confusion. For instance, on the concept of the motion of air molecules in a pipe, only about $17 \%$ of the students chose II (the correct answer), the rest either chose I, I and III, or II and III.

I. The air molecules oscillate perpendicular to the pipe.

II. The air molecules oscillate parallel to the pipe.

III. The air molecules move along the pipe in a sinusoidal curve.

If the textbooks that were shown to the students did not state explicitly that the amplitude of sound standing waves has no relation to the diameter of the pipe, then $88.1 \%$ of the students mistakenly interpreted, from the illustrations alone, that there is a relationship between the amplitude of sound standing waves and the diameter of a pipe. For example, students interpreted illustrations such as those shown earlier in Fig. 1 as follows: Fig. 1(a) the amplitude of sound standing waves exceeds the diameter of the pipe; Fig. 1(b), the amplitude of the sound standing waves is equal to or less than the diameter of the pipe; and Fig. 1(c), the amplitude of sound standing waves is less than the diameter of the pipe.

In addition, if the textbooks shown to the students only state nodes, but do not differentiate a pressure node from a displacement node or a pressure antinode from a displacement antinode, then students have no idea how the two differ from each other.

In contrast, illustrations that contained explanatory text had marked positive effects on student understanding of the motion of air molecules, the relationship between the amplitude of sound standing waves and the diameter of the pipe, and the difference between pressure nodes and displacement nodes or between pressure antinodes and displacement antinodes. For example, the following textbook excerpt (p. 523 in Ref. [9]) which states "The air molecules oscillate back and forth parallel to the direction in which the wave is traveling, creating compression (regions of higher pressure) and rarefactions (regions of 
lower pressure)," enhances student understanding of the motion of air molecules as demonstrated by approximately $78 \%$ of the students who correctly answered the question on air molecular motion.

\section{THEORETICAL FRAMEWORK}

Cognitive psychology and semiotics theories provide the framework for this research. From a cognitive psychology perspective, the concept of meaningful learning refers to the process of mentally connecting new concepts to an already existing cognitive structure through actively attending to relevant attributes of a phenomenon, and organizing and integrating these attributes into the existing cognitive structure [24-31]: for instance, relating sound standing wave patterns to already learned relevant concepts such as Dalton's molecular theory of gases, density and pressure, mechanism of energy transfer through an elastic medium, characteristics of waves, differentiation of transverse and longitudinal waves, the superposition of incident and reflecting waves, and the like.

So, how can one make scientific illustrations meaningful to the readers? One can make them meaningful through the use of proper symbols and explanatory text. Once the prerequisite cognitive structure has been established, research finds that illustrations accompanied by explanatory text improve student comprehension [32,33], whereas illustrations alone are not effective for student learning. For example, one study shows that an isoclines graph in ecology without adjunct explanative text is not effective for student learning [2].

Besides text, proper usage of symbols in an illustration influences the effectiveness of learning. Semiotics theory propounds that meaningful learning occurs when one can relate a symbol to the object to which it refers and connect the relevant aspects of the object to one's existing cognitive structure $[34,35]$. It suggests a triad relationship between a symbol, its object, and the mental association between a symbol and its object [36,37]. Hence, a symbol should, in this case, visually match some aspect relevant to the characteristic of the object it signifies in order to facilitate mental interpretation. For example, a sine wave is a good signifier for a transverse wave because it is relevant to the phenomena: a transverse wave causes a rope to move up and down in different regions as the wave propagates forward. Similarly, two intertwining sinusoidal waves are relevant to transverse standing waves because of their appearance on a resonating rope and, thus, are conventionally used to signify transverse standing waves. However, a sine wave is not, without a prerequisite cognitive structure, a meaningful signifier for sound waves, longitudinal pressure waves in nature where media oscillate back and forth as the waves propagate forward. Thus, in a sound standing wave, compression and rarefaction characteristics should determine the symbol or symbols we choose to illustrate it, so that the symbol or symbols are meaningful for the reader. One might illustrate a standing sound wave by dots densely packed in a defined area (compression) followed by dots much less densely packed in an equal area (rarefaction).

Based on the pilot study and the theoretical framework in cognitive psychology and semiotics, we set the objectives of this research as follows: (i) To find out how students interpret the illustrations for sound standing waves in air columns in pipes without detailed explanatory text in a typical introductory physics textbook; (ii) to design more effective illustrations with supporting text to improve student understanding of the underlying concepts; and (3) to test the hypotheses that the newly designed illustrations with their supporting text are more effective for student learning than the illustrations in a typical introductory physics textbook.

\section{MATERIALS AND METHODS}

\section{A. Participants}

During the 2011-2012 academic year, at a predominantly Hispanic institution in South Texas, thirty volunteers for research were recruited from the following introductory college physics courses: The first author contacted all the students (240) who had just completed General Physics I and Physics for Scientists and Engineers I courses. Out of the 240, 90 volunteered to participate in the research; however, only 30 qualified. These students had to meet two criteria-(i) they had completed the first course (which covered sound) of the two-course sequence of introductory physics courses, and (ii) they had answered, satisfactorily, a short questionnaire consisting of five questions (shown below) on the basic knowledge of sound waves and transverse standing waves: they had answered four out of the five questions correctly, and approximately $87 \%$ had either correctly or partially correctly answered the remaining question.

(1) What is a sound wave?

(2) Is a sound wave a longitudinal wave or a transverse wave? Explain your answer.

(3) Please draw a graphical representation of a sound wave and clearly label all parts of your drawing.

(4) What is a transverse standing wave? And how is a transverse standing wave formed?

(5) What are an antinode and a node in a transverse standing wave?

Prior to answering the five questions, access to a 1 hour online video containing demonstrations and explanations of periodic sound waves, transverse and longitudinal waves, and transverse standing waves provided all the participants refresher training. These procedures - first physics course completion, then video presentation, and then satisfactorily answering five questions-ensured that all the research volunteer participants had the basic knowledge of sound waves and transverse standing waves, two concepts that 
usually precede the sound standing wave phenomena in an introductory physics textbook.

Typically, volunteer students tend to be more able than students in general $[38,39]$. Therefore, the researchers believe that if the volunteers have problems with the traditional textbook illustrations, then the less able students will surely have problems.

\section{B. Instruments}

Five instruments were employed in this study: one "Presurvey", two handouts labeled "Textbook Handout" and "Another Perspective," and two postsurveys labeled "Post Alt" and "Post Text."

\section{Presurvey}

The Presurvey (see Appendix A) consists of 14 multiple choice questions targeting three conceptual areas about which students often feel confused: (i) the motion of air molecules inside a pipe (questions 2, 9-14); (ii) the relationship between the amplitude of sound standing waves and the diameter of the pipe (question 3); and (iii) the pressure and displacement nodes and antinodes in open-open and open-closed pipes (questions 4-8). These questions are based on the researchers' findings in the prior pilot study concerning student understanding of sound standing waves $[22,23,40]$.

\section{Textbook Handout}

The Textbook Handout is an excerpt from a textbook [11] currently used for the General Physics course. It contains diagrams for sound standing waves represented by two intertwining sinusoidal curves (see Appendix B). This Textbook Handout is one of the other five textbooks [8-12] commonly used in colleges and universities. They all contain the illustrations conventionally used for transverse standing waves to signify the longitudinal standing waves phenomena in both open-open and open-closed tubes. Furthermore, these illustrations do not have explanatory text accompanying them except in one textbook by Knight [9] on the case of second harmonics in an open-open tube only.

Furthermore, only two of the five textbooks state that there is no relationship between the amplitude of sound standing waves and the diameter of the pipe. The other three have no mention of the concept. Only two textbooks make distinctions between pressure nodes or antinodes and displacement nodes or antinodes; the other three do not. Three out of five state how air molecules move inside the pipe in general; the other two do not.

\section{Another Perspective}

The Another Perspective handout (see Appendix C), mainly designed by the first author, contains illustrations of the motion of air molecules with supporting text for sound standing waves in a pipe (see a sample illustration in Fig. 2). These illustrations are in alignment with the mathematical equations for sound standing waves in cylindrical pipes described in books on acoustics [41-45]. They also adhere to conventions in designing instructional graphics such as arrows, graphs, labels, and the like [46]. In addition, they adhere to the embodiment of sequential operation processes in illustrations to show how scientific devices work [47].

Four distinguishing characteristics of these illustrations were developed for the motion of air molecules in pipes: (i) the essential element of the motion-air molecules represented by dots, (ii) the main sequential phases in a periodic motion cycle, (iii) the arrows and labels that further facilitate the interpretation of the motion of air molecules to identify compression and rarefaction zones and to locate pressure and displacement nodes and antinodes, and (iv) the separate pressure and displacement graphs, outside the pipes.

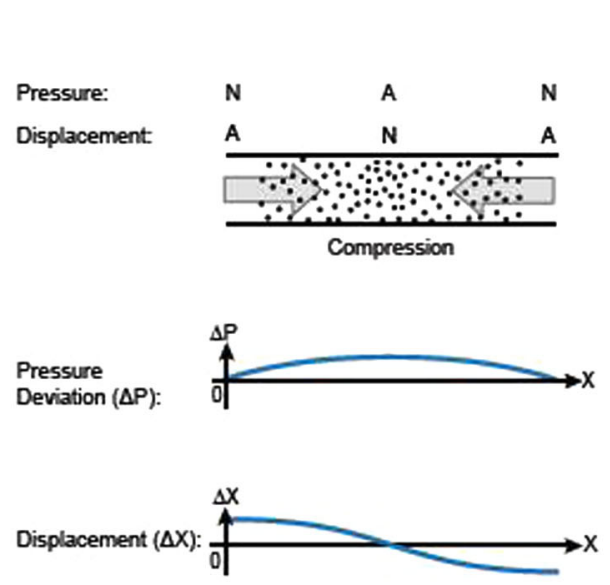

(a) At one moment
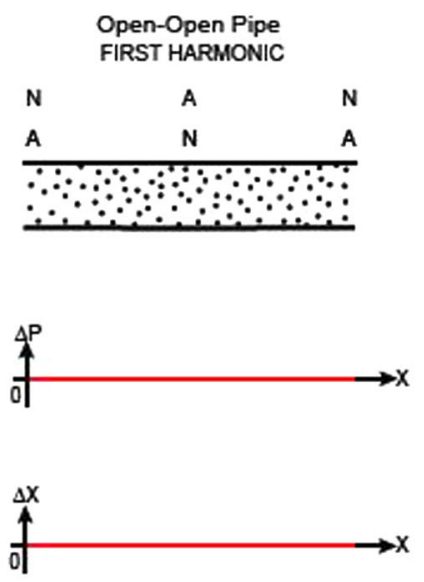

(b) A quarter cycle later than (a)
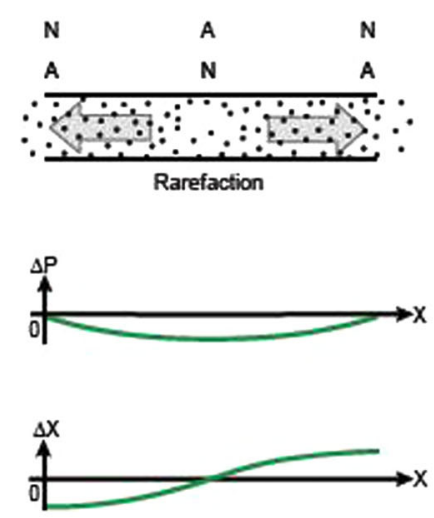

(c) A half cycle later than (a)

FIG. 2. First harmonic sound standing wave in an open-open pipe. 
In addition, explanatory text was provided for the illustrations. Furthermore, refutation text was added to deal with common student misinterpretations of figures [48]. An example of refutation text is "the amplitude of the displacement or pressure curves $\ldots$ is not related to diameter of the pipe." There is no refutation text in the Textbook Handout. The textbook published by Knight [9] has the statement on page 525 .

\section{Two postsurveys: Post Alt and Post Text}

The Post Alt and Post Text (see Appendix D) read exactly the same as the presurvey except that each includes a new question (question 3) that refers to specific figures; however, the figure numbers differ due to the different handouts. Also, both postsurveys include spaces for students to record their reasoning and basis or source(s) of information in coming up with their answer for each question. Sources may have included specific page(s) in the text of the handout, specific figure numbers in the handout, previous knowledge, or demonstrations observed in class.

\section{Experimental design}

In order to test the effects of the two handouts and collect students' feedback as to why they think a particular handout enhances their understanding, each student received two treatments, i.e., reading two different handouts in the order indicated in Fig. 3. Further, to eliminate any sequential effect that reading the first handout might have on reading the second handout, this research employs a counterbalanced design [49], in which two groups read different initial handouts and later in the process read the opposite handouts.

\section{Procedures}

Thirty volunteer students were randomly assigned to two groups of fifteen each - group A and group B. A random number generator in Microsoft Excel 2010 generating either a number "1" (group A) or "2" (group B) determined the group membership of each student.

Once group membership was determined, the administration procedures took place in the following order: The Presurvey was administered to all the participants to gauge their initial understanding of sound standing waves; they were given approximately 30 minutes to complete the survey without being given any handouts to read. Further, they were orally instructed to answer the questions as best they could, based on their current knowledge. Following the Presurvey, each student read an assigned handout: group A read Another Perspective, while group B read the Textbook Handout. Using the handout, group A completed Post Alt while group B completed Post Text. This took about 45 minutes. Then, the procedure was reversed: group A read the Textbook Handout while group B read Another Perspective. It also took approximately 45 minutes to

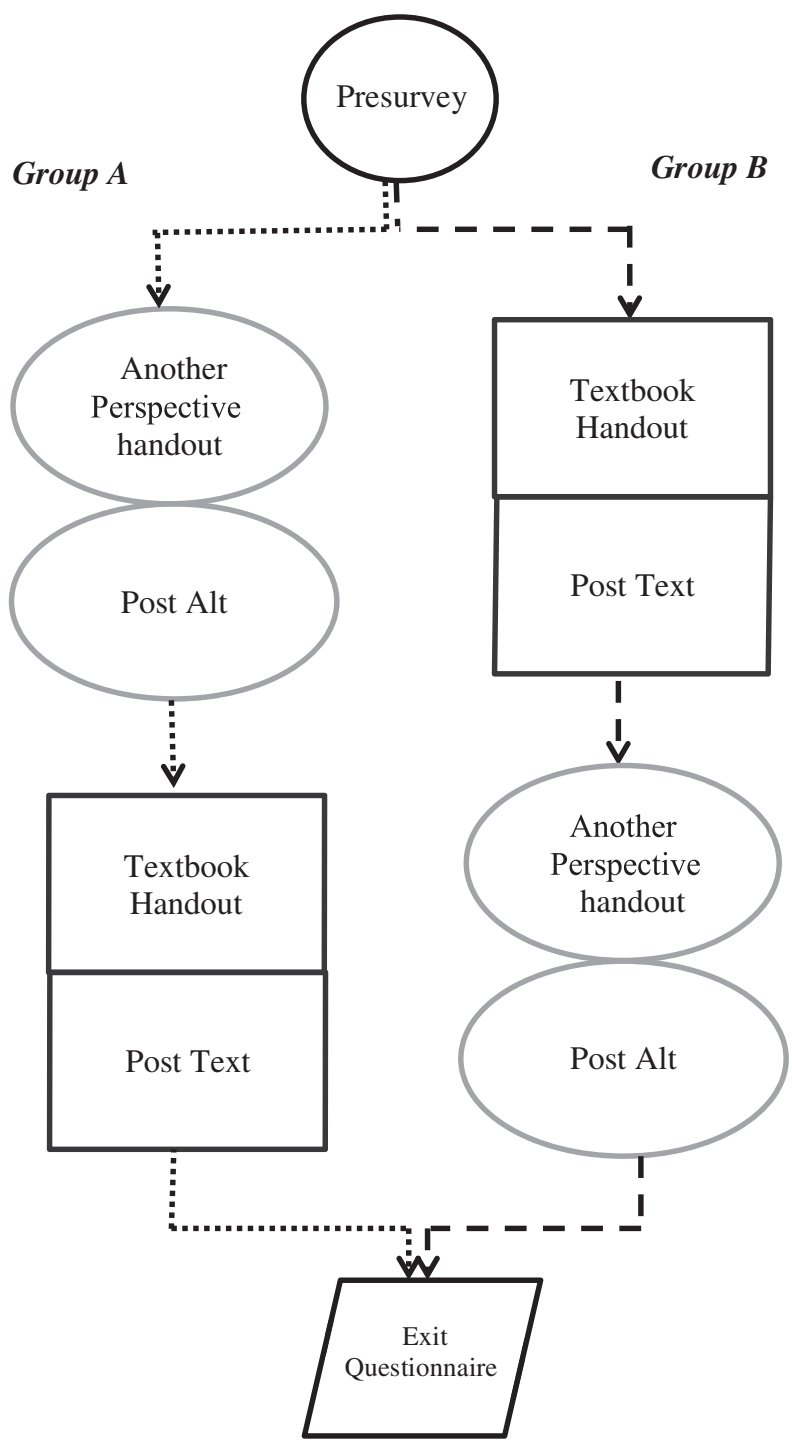

FIG. 3. Flow chart of activities for groups A and B.

complete the second postsurvey, but this time group A completed Post Text while group B completed Post Alt. Finally, all participants answered a questionnaire which contained three open-ended questions regarding the comparative effectiveness of the two handouts.

\section{E. Statistical methods}

This study compares either mean scores or proportions. In all the mean score comparisons, the KolmogorovSmirnov goodness-of-fit test assesses the normality of the distributions. An independent samples case $t$ test is used when comparing mean scores of two groups and if the difference scores are normally distributed; otherwise, an independent sample Mann-Whitney $U$ test is used. However, when comparing the mean scores of a single group on two surveys and when the difference scores are 
normally distributed, a paired samples case $t$ test is used; otherwise, a paired Wilcoxon signed-rank test is used.

On the other hand, when comparing the proportions between two groups, a two proportions $Z$ test is used. However, when comparing a proportion for one group with the proportion 0.5 , a one-proportion $Z$ test is used. The significant level of all tests is set at 0.05 . The software of choice are SPSS 20.0 for Windows and JMP 8.0.

The research took place in the following order: First, the researchers compared the means of the overall scores of the two groups in the presurvey to determine whether the groups differed significantly in prior knowledge on this topic. Second, for each group, they tested for a significant difference between the presurvey and the first postsurvey (Post Alt for group A, Post Text for group B) scores. Third, they tested for a significant difference between the second postsurvey scores (Post Text for group A, Post Alt for group B) and the first postsurvey scores for each group. This procedure was repeated for each of the three conceptual areas embedded in the surveys: (i) the motion of air molecules, (ii) the relationship between the amplitude of sound standing waves and the diameter of pipe, and (iii) the pressure and displacement nodes and antinodes. Finally, they tallied the student responses to the questionnaire and tested whether a majority favored a particular handout.

The null hypotheses are as follows:

(1) On the motion of air molecules in a pipe, there is no difference in gain scores between the group that reads the Another Perspective handout and the group that reads the Textbook Handout.

(2) On the relationship between the amplitude of sound standing waves in a pipe and the diameter of the pipe, there is no difference in gain scores between the group that reads the Another Perspective handout and the group that reads the Textbook Handout.

(3) On the difference between pressure nodes and antinodes and the difference between displacement nodes and antinodes in a pipe, there is no difference in gain scores between the group that reads the Another Perspective handout and the group that reads the Textbook Handout.

\section{RESULTS AND DISCUSSIONS}

This section shows the results of an overall analysis of the data. It further analyzes student gain scores in each of the three following concept areas: the motion of air molecules, the relationship between amplitude of sound standing waves and diameter of pipe, and the pressure and displacement nodes and antinodes.

Descriptive statistics on presurvey and postsurvey scores for two groups are shown in Table I. One point is assigned to each correct answer.

Comparing the means of presurvey scores for group A (4.93) and group B (4.33) through an independent samples Mann-Whitney $U$ test, we conclude that each group's initial
TABLE I. Descriptive statistics on overall survey scores for groups $\mathrm{A}$ and $\mathrm{B}$ ( $n=15$ for each group).

\begin{tabular}{lcrr}
\hline \hline Group & Survey & $\bar{x}^{*}$ & Median \\
\hline A & Pre & $4.93 \pm 0.68$ & 5.00 \\
& Post Alt & $11.00 \pm 0.58$ & 11.00 \\
& Post Text & $10.47 \pm 0.80$ & 10.00 \\
B & Pre & $4.33 \pm 0.45$ & 4.00 \\
& Post Text & $4.40 \pm 0.41$ & 4.00 \\
& Post Alt & $9.60 \pm 0.67$ & 10.00 \\
\hline \hline
\end{tabular}

*Data shown as mean \pm standard error of the mean.

knowledge of sound standing waves is not significantly different from the other.

Figure 4 displays boxplots for score distributions of presurvey and postsurvey scores for group A and group B. Post Alt and Post Text scores read higher than Presurvey scores in group A, and Post Alt scores read higher than both Presurvey and Post Text scores in group B.

Furthermore, testing for sequential effects, the overall mean of the two Post Alt scores is $10.3[(11.0+9.6) / 2]$, which is greater than the overall mean of the two Post Text scores, $7.44[(10.47+4.4) / 2]$. The related-samples Wilcoxon signed-rank test shows that the two overall means are significantly different $(P$ value $=0)$. These clearly show that the Another Perspective handout is superior to the Textbook Handout for both groups regardless of sequence.

In Table II, we tested the significance of gain scores between the means of the presurvey and postsurveys for each group. The means of total points on presurvey and postsurveys are compared using the paired Wilcoxon signed-rank test because the assumption of normality on the gain scores in each group does not hold, except for the difference between the Post Text and Post Alt scores for group A-in that case, a paired $t$ test was conducted. Significant differences in gain scores are found between the Post Alt and Presurvey for group A, as well as between Post Alt and Post Text for group B. These results indicate

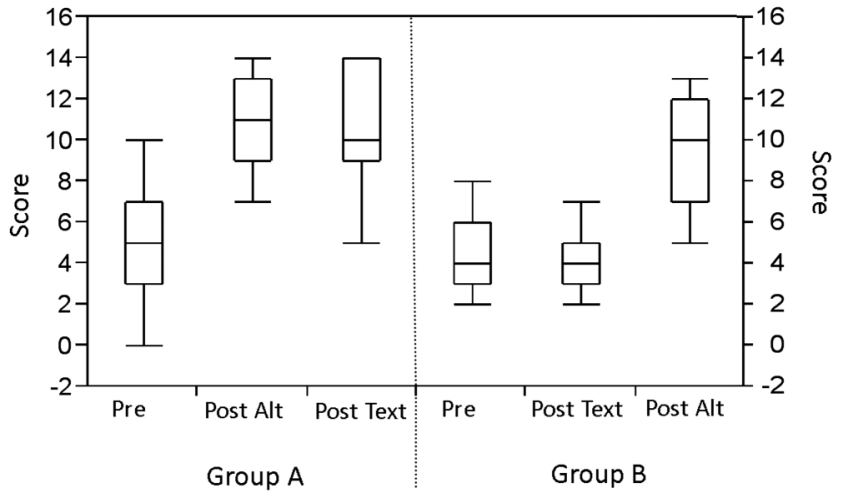

FIG. 4. Boxplots of score distributions: Presurvey, Post Alt, and Post Text for groups A and B. 
TABLE II. Overall gain scores between two surveys in comparison ( $n=15$ for each group).

\begin{tabular}{lcrll}
\hline \hline Group & Pair differences & \multicolumn{1}{c}{$M_{d}{ }^{*}$} & Median & $P$ value \\
\hline A & Post Alt-Pre & $6.07 \pm 0.84$ & 8.00 & 0 \\
& Post Text-Post Alt & $-0.53 \pm 0.38$ & 0 & 0.13 \\
B & Post Text-Pre & $0.07 \pm 0.69$ & 0 & 0.92 \\
& Post Alt_Post Text & $5.20 \pm 0.89$ & 6.00 & 0 \\
\hline \hline
\end{tabular}

*Data shown as mean of the score differences \pm standard error of the mean of the score differences.

that after students had read the Another Perspective handout, their understanding improved.

Regarding the student free responses in the Presurvey, most of the answers are either blank or incorrect. For instance, in responding to the question "Which of the following best describes a sound standing wave?" a student response clearly shows confusion: "I learned that sound waves are longitudinal. However, there is [sic] also transverse sound standing waves. (video). Therefore, I think they could be either longitudinal or transverse." We note that our online refresher video does not show transverse sound standing waves; rather, it shows transverse standing waves on a string.

In their free-response explanations in the postsurveys, the students in group A mainly refer to the figures such as Fig. 1, Fig. 2, or use terms such as compression and rarefaction that are found in the Another Perspective handout to support their answers for both postsurveys. Group B students at first support their answers by referring to the learning materials in the Textbook Handout. However, after reading the Another Perspective handout, they mainly support their answers similarly to the students in group A. For instance, to the question "In the open-open pipe, when the first harmonic sound standing wave is formed, which best describes the motion of the air molecules?" a typical response is as follows: "pressure antinode is formed where air molecules bunch together in the middle, where there is maximum pressure. Then air molecules spread apart. This shows that air pressure and density is decreasing." These phenomena indicate that the illustrations in the Another Perspective handout are meaningful to them since they keep referring to them.

\section{A. Concept area 1: Air molecule motion}

Students' scores are calculated for question 2 and questions 9-14 in the Presurvey (see Appendix A) and for question 2 and questions $10-15$ in each of the postsurveys (Post Text, Post Alt); (see Appendix D). Table III shows the gain scores. The Wilcoxon signed-rank test is used to calculate the gain scores between Post Text and Post Alt for group A. All other gain scores are calculated using paired $t$ tests. The two significant $P$ values indicate that students who read the Another Perspective handout make significant gains in their basic understanding of the motion
TABLE III. The motion of air molecules in sound standing waves: Gain scores for group A and group B $(n=15$ for each group).

\begin{tabular}{lcrll}
\hline \hline Group & Pair differences & \multicolumn{1}{c}{$M_{d}{ }^{*}$} & Median & $P$ value \\
\hline A & Post Alt-Pre & $3.47 \pm 0.76$ & 4.00 & 0 \\
& Post Text-Post Alt & $0 \pm 0.20$ & 0 & 1.00 \\
B & Post Text-Pre & $0.53 \pm 0.50$ & 0 & 0.30 \\
& Post Alt-Post Text & $2.60 \pm 0.58$ & 2.00 & 0 \\
\hline \hline
\end{tabular}

*Data shown as mean of the score differences \pm standard error of the mean of the score differences.

of air molecules in sound standing waves in pipes for the first three harmonics. These gains occur whether the Another Perspective handout is read after they take the Presurvey or after they read the Textbook Handout.

Regarding the motion of air molecules in sound standing waves, these results support the decision to reject the null hypothesis that there is no difference in gain scores between the group that read the Another Perspective handout and the group that read the Textbook Handout.

\section{The transition in student understanding of the motion of air molecules in sound standing waves}

The four motion types for the movement of air molecules in a sound standing wave are described as follows (see Appendix A, question 10 of the Presurvey, as an example): These four motion types are also presented in each of the postsurveys in the same sequence.

A.... air molecules move along one of the two sinusoidal curves while oscillating parallel to the pipe....

B.... air molecules move along one of the two sinusoidal curves while oscillating perpendicular to the pipe....

C.... air molecules compress toward the left pressure antinode and reduce density and pressure around the right pressure antinode....

D.... air molecules oscillate up and down within the bounded areas of the two sinusoidal curves while propagating along the pipe....

Each of the six questions on the Presurvey, Post Text, and Post Alt surveys has four selection type distractors (A, B, C, D) and all have one correct answer (C). Figure 5 illustrates the percentage of the students who selected different distractors on each survey: for instance in group A, the Presurvey, only $13 \%$ of the students (two students) chose the correct answer for all six questions. It is also apparent that the other $87 \%$ of the students chose one, two, or three different distractors in answering all six questions. In contrast, group A, Post Alt shows that $80 \%$ of the students chose only the correct answer in each of the six questions.

Note that in group A the Another Perspective handout changes the student perception of how air molecules move in sound standing waves as evidenced by the persistence of 
Group A: Presurvey

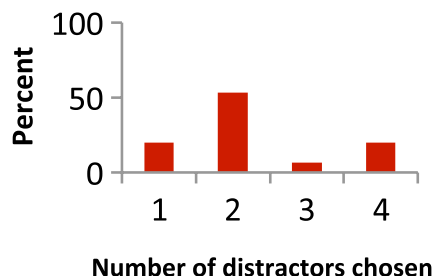

(a)

Group B: Presurvey

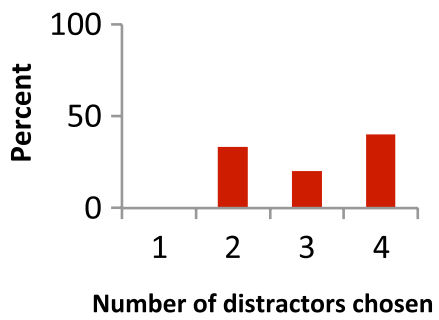

(d)
Group A: Post Alt

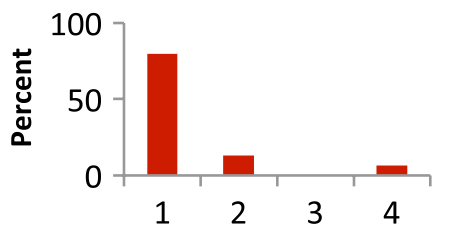

Number of distractors chosen

(b)

Group B: Post Text

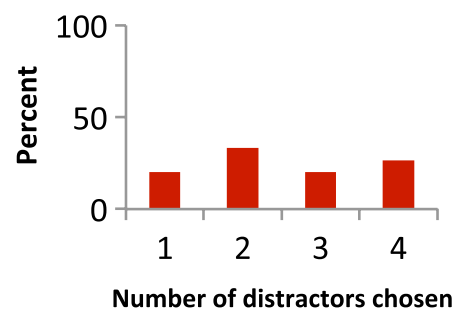

(e)
Group A: Post Text

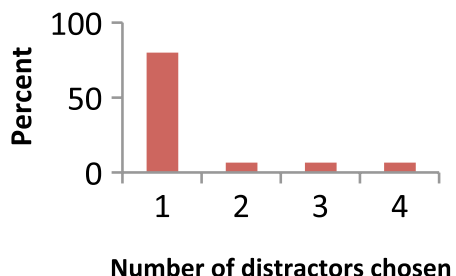

(c)

Group B: Post Alt

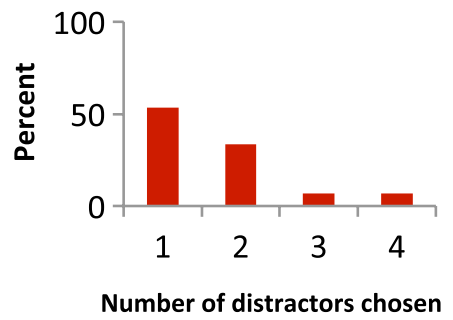

(f)

FIG. 5. The percentage of students who chose either one, two, three, or four distractors in questions 9-14 in the Presurvey and in questions 10-15 in the Post Alt and Post Text surveys for group A and group B.

this perception even after they read the Textbook Handout. Similar changes in perception occur in group B: $6.65 \%$ (only one student) of the group B students consistently makes the correct choice after reading the Textbook Handout; however, after reading the Another Perspective handout, $53.33 \%$ consistently make the correct choice.

Reflection on the diversity of answers to questions regarding the motion of air molecules for different harmonics in both an open-open pipe and an open-closed pipe reveals great conceptual confusion about how air molecules move. For example, some think that air molecules literally move along the curves - the narrower regions between the curves (nodes) indicate that pressure increases, whereas the wider regions (antinodes) indicate that pressure decreases.

\section{B. Concept area 2: Relationship between the amplitude of sound standing waves in a pipe and the pipe's diameter}

Question 4 reads as follows: How does the amplitude of the sound standing waves relate to the diameter of the pipe in your opinion?

A. The amplitude of the sound standing waves is greater than the diameter of the pipe.

B. The amplitude of the sound standing waves is smaller than the diameter of the pipe.

C. The amplitude of the sound standing waves is equal to the diameter of the pipe.

D. The amplitude of the sound standing waves is not at all related to the diameter of the pipe.
From Fig. 6(a), one can see that $20 \%$ of the students in each group chose the right answer D, whereas the remaining $80 \%$ believed there is some relationship between the amplitude of the sound standing waves and the diameter of the pipe. However, after reading the Another Perspective handout, more than $70 \%$ of the group A students changed to the correct answer. These students still consistently chose the right answer, even after they read the Textbook Handout. In contrast, about $70 \%$ of group B students chose the wrong answers after reading the Textbook Handout, their first postsurvey. However, after reading the Another Perspective handout, about $53 \%$ of them changed to the correct answer.

To quantify the effects of the Another Perspective handout in bringing about changes in student understanding of the relationship between the amplitude of a sound standing wave and the diameter of a pipe, we conducted two-proportions $Z$ tests on the changes from wrong answers to correct answers between the Presurvey and the first postsurvey and between the first postsurvey and the second postsurvey, respectively.

Eight students in group A and two students in group B changed from a wrong answer in the Presurvey to the right one in the first postsurvey (see Fig. 7).

Utilizing the two-proportions $Z$ test [50],

$$
z=\frac{x_{A} / n_{A}-x_{B} / n_{B}}{\sqrt{\hat{p}_{P}\left(1-\hat{p}_{P}\right)} \sqrt{1 / n_{A}+1 / n_{B}}},
$$

where $x_{A}$ and $x_{B}$ represent the change in the number of correct answers out of two groups, $n_{A}$ and $n_{B}$, respectively; 


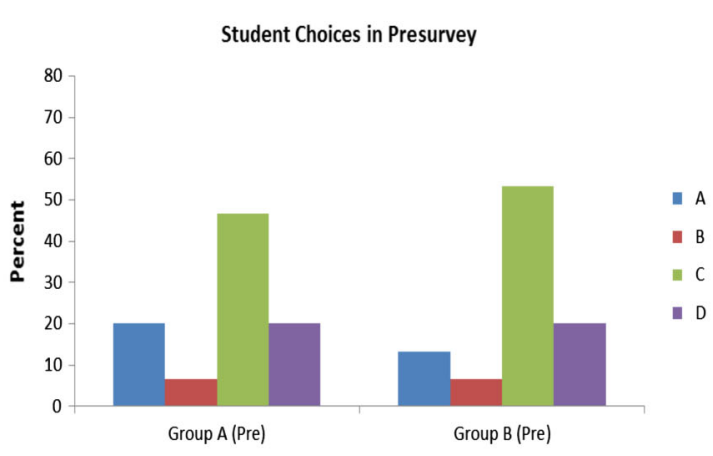

(a)

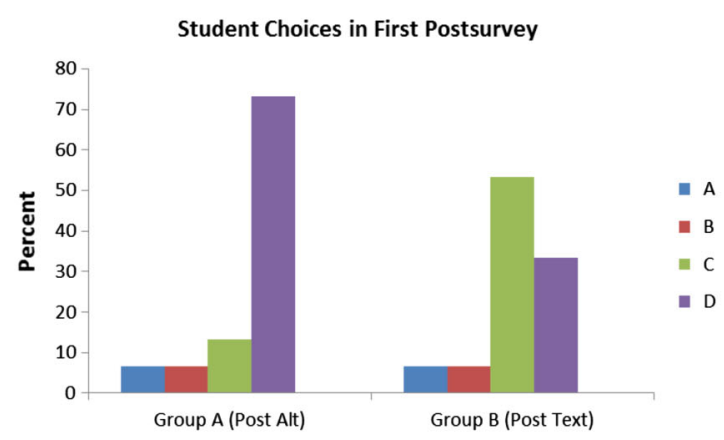

(b)

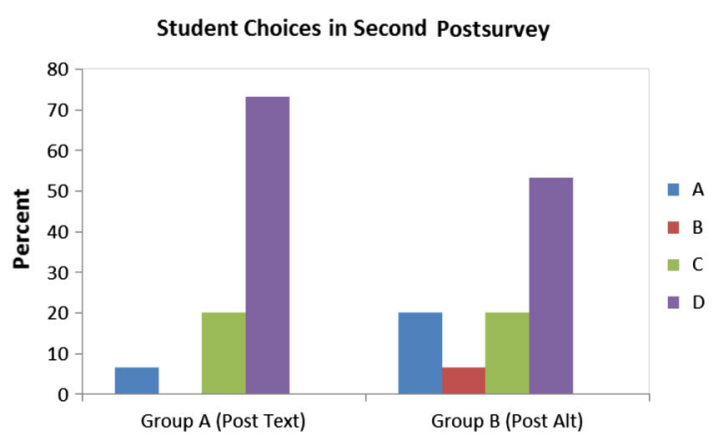

(c)

FIG. 6. Percentages of students in group A and group B choosing options A through D in question 3 in the Presurvey and question 4 in the first and second postsurveys.

$\hat{p}_{P}$ is the pooled sample proportion which is calculated as $\hat{p}_{P}=\left(x_{A}+x_{B}\right) /\left(n_{A}+n_{B}\right)=(8+2) /(15+15)=0.33$, the test statistic is

$$
z=\frac{\frac{8}{15}-\frac{2}{15}}{\sqrt{0.33(1-0.33)} \sqrt{\frac{1}{15}+\frac{1}{15}}}=2.32 \quad(P \text { value }=0.01)
$$

From this result one can infer that group A has a significantly greater increase in understanding this

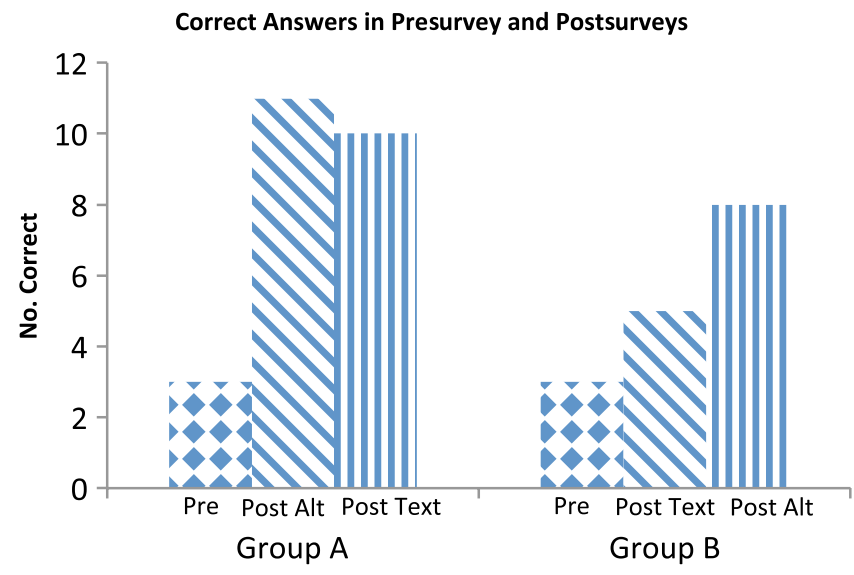

FIG. 7. Number of correct answers in the Presurvey and postsurveys. relationship from the Presurvey to the first postsurvey, compared to group B.

Utilizing the two-proportions $Z$ test, again, to compare the changes of wrong to correct answers between the first postsurvey and the second postsurvey for the two groups, $x_{A}$ and $x_{B}$ are now equal to 0 and 3 , respectively. Since the pooled proportion $\hat{p}_{P}=\left(x_{A}+x_{B}\right) /\left(n_{A}+n_{B}\right)=$ $(0+3) /(15+15)=0.1$, the test statistic becomes

$z=\frac{\frac{0}{15}-\frac{3}{15}}{\sqrt{0.1(1-0.1)} \sqrt{\frac{1}{15}+\frac{1}{15}}}=-1.83 \quad(P$ value $=0.03)$.

This result indicates that group B also has a significant increase in understanding the relationship after reading the Another Perspective handout, whereas group A, who read the Textbook Handout, has no significant change.

\section{Concept area 3: Pressure and displacement nodes and antinodes}

Questions 5-9 in Post Alt and Post Text, and questions 4-8 in the Presurvey pertain to the displacement and pressure antinodes and nodes in a sound standing wave in an air column inside a pipe. Since each question counts one point, the maximum number of points is five. Paired 
TABLE IV. Gain scores on concept of pressure and displacement nodes and antinodes ( $n=15$ for each group).

\begin{tabular}{lcrcc}
\hline \hline Group & Pair differences & \multicolumn{1}{c}{$M_{d}{ }^{*}$} & Median & $P$ value \\
\hline A & Post Alt-Pre & $1.80 \pm 0.33$ & 1.00 & 0.001 \\
& Post Text-Post Alt & $-0.47 \pm 0.34$ & 0 & 0.196 \\
B & Post Text-Pre & $-0.93 \pm 0.30$ & -1.00 & 0.008 \\
& Post Alt-Post Text & $2.40 \pm 0.50$ & 3.00 & 0.002 \\
\hline \hline
\end{tabular}

*Data shown as mean of the score differences \pm standard error of the mean of the score differences.

Wilcoxon signed-rank tests were conducted for all the pairs, except between Post Text and Presurvey for group B where the paired $t$ test was conducted.

Table IV shows that there is a significant gain between the Presurvey and Post Alt for group A, which shows that the Another Perspective handout is effective in improving student understanding of these concepts. In contrast, a significant loss is found between the Presurvey and Post Text for group B, which shows that the Textbook Handout is detrimental to improving student understanding of these concepts: most students confused pressure nodes with displacement nodes and pressure antinodes with displacement antinodes. However, reading the Another Perspective handout after the Post Text significantly helped group B improve their understanding as evidenced by a significant positive gain between Post Alt and Post Text.

\section{Exit questionnaire}

All twenty-nine students were surveyed at the same time. The questionnaire consisted of three questions: 1 . What do you think about the two handouts? 2. Which one makes you understand better? 3. Which pictorial representations make you understand sound standing waves better and why?

In the question, "What do you think about the two handouts?" almost all the students think that the Another Perspective handout is more informative and easier to understand than the Textbook Handout. Some students specifically commented that the Textbook Handout is confusing.

In the question, "Which handout makes you understand better?" twenty-seven students strongly favored the Another Perspective handout, and two students favored the Textbook Handout. One student liked both handouts. The proportion favoring the Another Perspective handout is $0.90(=27 / 30)$. The one-proportion $Z$ test [51] shows that students claim to attain a better understanding with the Another Perspective handout,

$$
z=\frac{\hat{p}-p_{0}}{\sqrt{p_{0}\left(1-p_{0}\right) / n}}
$$

$\hat{p}$ is the proportion of those who comment that the Another Perspective handout makes them understand better, which can be calculated as $\hat{p}=x / n=\frac{27}{30}=0.90 ; p_{0}=0.50$. Consequently, the value of the test statistic is

$$
z=\frac{0.90-0.50}{\sqrt{0.50(1-0.50) / 30}}=4.38 \quad(P \text { value }=0) .
$$

Student comments on why the Another Perspective handout made them understand better include the following:

- "I was able to understand the movement of the air molecules due to the figures drawn. It was easy for me to read and understand the process."

- "The material was explained clearly and the figures for each harmonic were very useful! It cleared up any misunderstanding I had."

- "Helped me better understand because at first the textbook copy said an open end has an antinode. However, I learned through the Another Perspective handout that an open end pipe has a pressure node and a displacement antinode."

- "It used visuals more and described how the pressure and displacement are related. The Textbook Handout only gave a bit description about the pressure. It was more about the displacement. At one point I had trouble figuring out which visual representation of the $\sin$ wave was pressure and which one was displacement."

- "I was able to remember the figures from it to answer the questions."

In the question, "Which pictorial representations make you understand sound standing waves better and why?" twenty-four students stated that the figures in the Another Perspective handout made them understand better, and five students commented that the figures in the Textbook Handout made them understand better. One student stated that they were equally helpful in helping to understand. The proportion of students who stated the illustrations in the Another Perspective handout helped them understand better is $0.80(=24 / 30)$. Based on Eq. (2),

$$
z=\frac{0.80-0.50}{\sqrt{0.50(1-0.50) / 30}}=3.29 \quad(P \text { value }=0.0005)
$$

The data provide sufficient evidence to conclude that a majority of the students favored the figures in the Another Perspective handout.

Furthermore, students commented on the illustrations in the Another Perspective handout. Specifically, 53\% of the students made comments on the processes aspect of the illustrations such as "More useful for my understanding because it showed the compression and rarefaction of air molecules," and "Before seeing those pictures, I thought the 
air molecules were moving up or down (perpendicular) to the pipe, but they are actually parallel."

Thirty percent of the students remarked that the elements including the particles, labels, and arrows included in the illustrations in the Another Perspective handout helped with their understanding. For example, a student mentioned, "I like the arrows that helped me understand the compression of the air molecules. The letter on top with the proper names, also were very helpful in understanding which were the nodes and antinodes. The scattered dots helped me visualize the air molecules much better."

Finally, $13.33 \%$ of the students noted that the distinction between pressure and displacement standing wave graphs and the separation between the pipe and the pressure and displacement standing waves on the graphs helped with their understanding. Sample comments included, "It also distinguished between pressure and displacement in the graphs. Just by looking at them helps you understand the text. The chapter given to us from the textbook used hard to understand pictorial representations that were not explained well. It bothered me that they did not inform us it was showing displacement not pressure." Another student said, "I like that it had the air molecules and displacement graphs separately. It gave me a better understanding of the standing wave. I did, however like the A, N, A pattern sequence on the textbook version. It seems like it would help with memorizing."

From perusing all the student comments, we note that students prefer the illustrations clearly laying out the processes through incorporating elements, arrows, and labels. We find overwhelming evidence that they can easily relate the dots in the illustrations to air molecules: varying the density of the dots corresponds to varying the air pressure at the pressure antinodes. Because air molecules, density, and pressure are concepts that already exist in their cognitive structure, they can more readily understand the formation of pressure antinodes in sound standing waves.

\section{CONCLUSIONS}

Our study investigated difficulties that students experience with interpreting the illustrations of sound standing waves in existing introductory physics textbooks. We found that they cannot interpret, correctly, the motion of air molecules in sound standing waves from the illustrations depicting two-intertwining sine curves; the typical transverse standing wave illustrations used to convey longitudinal standing wave phenomena are just not meaningful to them. In addition, students cannot make a meaningful link between illustrations of sound standing waves and the underlying physics due to the lack of explanative text accompanying the illustrations.

Our research shows that the new design consisting of illustrations accompanied by explanative text is highly effective in facilitating student learning of the physics of sound standing waves. It reflects the suggestions from semiotic and cognitive psychology theories in terms of the necessary components in an effective illustration. These components include air molecules, sequential processes in a cycle of the wave motion, and arrows and labels that clarify the motion. Student responses to our questionnaires reveal that the new design helps them to visualize the processes and to connect what they are learning to what they already know in a meaningful way.

Although the sample consists of volunteers, in all likelihood, the new illustrations and supporting text will also benefit the student population in general in introductory physics courses. This needs to be confirmed by further research with the general student population. We recommend that textbook publishers consider improving the traditional textbook illustrations by referring to the new design for sound standing waves. We also recommend that physics instructors ensure that students attain the prerequisite cognitive structure necessary to make learning of sound standing waves meaningful. Finally, we recommend that in presenting new illustrations, diagrams, and graphs, instructors provide sufficient elaborative explanations to students in order to avoid misinterpretations.

\section{ACKNOWLEDGEMENTS}

This project was funded by the Undergraduate Research Initiative Mini-Grants 2008-2012 at the University of Texas-Pan American.

\section{APPENDIX A: PRESURVEY}

Instruction: After bubbling in your answer for each question on your Scantron, please provide your reason, basis, or source for your answer to each question on the blank line. Sources may include, for example, specific page(s) in the text of the handout, specific figure numbers in the handout, or your previous knowledge.

1. Which of the following best describes a sound standing wave?

A. It is a longitudinal wave.

B. It is a transverse wave.

C. Sometimes transverse sometimes longitudinal.

D. It is an electromagnetic wave.

2. Which of the following is TRUE about the motion of the air molecules when a sound standing wave is formed in an air column inside a pipe?

I. The air molecules oscillate perpendicular to the pipe.

II. The air molecules oscillate parallel to the pipe.

III. The air molecules move along the pipe in a sinusoidal curve. 
A. I only, B. II only, C. III only, D. I \& III, E. II \& III.

3. How does the amplitude of the sound standing waves relate to the diameter of the pipe in your opinion?

A. The amplitude of the sound standing waves is greater than the diameter of the pipe.

B. The amplitude of the sound standing waves is smaller than the diameter of the pipe.

C. The amplitude of the sound standing waves is equal to the diameter of the pipe.

D. The amplitude of the sound standing waves is not at all related to the diameter of the pipe.

4. Which of the following is TRUE about the displacement antinode in a sound standing wave in an air column inside a pipe?

A. The displacement antinode depicts where the displacement of air molecules can have the largest variation from the equilibrium position in a direction parallel to the pipe.

B. The displacement antinode depicts where the displacement of air molecules from the equilibrium position is always at a maximum in a direction parallel to the pipe.

C. The displacement antinode depicts where the displacement of air molecules can have the largest variation from the equilibrium position in a direction perpendicular to the pipe.

D. The displacement antinode depicts where the displacement of air molecules from the equilibrium position is always at a maximum in a direction perpendicular to the pipe.

5. Which of the following is TRUE about the pressure node and pressure antinode in a sound standing wave in an air column inside a pipe?

I. The pressure node in a sound standing wave is where the air pressure is zero.
II. The pressure node in a sound standing wave is where the air pressure has no deviation from atmospheric pressure.

III. The pressure antinode in a sound standing wave is where the air pressure is always at a maximum.

IV. The pressure antinode in a sound standing wave is where the air pressure has the largest variation between a maximum compression (air pushing together) and a maximum rarefaction (air pulling apart).

A. I and III, B. I and IV, C. II and III, D. II and IV.

6. Which of the following is TRUE about a sound standing wave in an air column inside a pipe with two open ends?

I. The open ends of the air column are pressure nodes.

II. The open ends of the air column are pressure antinodes.

III. The open ends of the air column are displacement nodes.

IV. The open ends of the air column are displacement antinodes.

A. I and III, B. I and IV, C. II and III, D. II and IV.

7. Which of the following is TRUE about a sound standing wave in an air column inside a pipe with one closed end and one open end?

I. The open end of the air column is a pressure node.

II. The open end of the air column is a pressure antinode.

III. The open end of the air column is a displacement node.

IV. The open end of the air column is a displacement antinode.

A. I and III, B. I and IV, C. II and III, D. II and IV.

8. Which of the following is TRUE about a sound standing wave in an air column inside a pipe with one closed end and one open end? 
I. The closed end of the air column is a pressure node.

II. The closed end of the air column is a pressure antinode.

III. The closed end of the air column is a displacement node.

IV. The closed end of the air column is a displacement antinode.

A. I and III, B. I and IV, C. II and III, D. II and IV.

9. In an open-open pipe, when the first harmonic sound standing wave is formed, which best describes the motion of the air molecules?

A. At one moment, air molecules move along one of the two sinusoidal curves while oscillating parallel to the pipe. A half cycle later, air molecules move along the other sinusoidal curve while oscillating parallel to the pipe.

B. At one moment, air molecules move along one of the two sinusoidal curves while oscillating perpendicular to the pipe. A half cycle later, air molecules move along the other sinusoidal curve while oscillating perpendicular to the pipe.

C. At one moment, air molecules compress towards the center of the pipe. A half cycle later, air molecules reduce pressure and density around the center of the pipe and extend themselves towards both ends.

D. During the entire cycle, air molecules oscillate up and down within the bounded areas defined by the two sinusoidal curves while propagating along the pipe. As time progresses, the two curves grow narrower around the center and then pull apart, indicating air pressure increases and then decreases accordingly, with the narrower regions associated with higher pressure and the wider regions associated with lower pressure.

E. None of the above. (Please state your answer here:
10. In an open-open pipe, when the second harmonic standing wave is formed, which best describes the motion of the air molecules?

A. At one moment, air molecules move along one of the two sinusoidal curves while oscillating parallel to the pipe. A half cycle later, air molecules move along the other sinusoidal curve while oscillating parallel to the pipe.

B. At one moment, air molecules move along one of the two sinusoidal curves while oscillating perpendicular to the pipe. A half cycle later, air molecules move along the other sinusoidal curve while oscillating perpendicular to the pipe.

C. At one moment, air molecules compress towards the left pressure antinode and reduce density and pressure around the right pressure antinode. A half cycle later, air molecules reduce density and pressure around the left pressure antinode and compress around the right pressure antinode.

D. During the entire cycle, air molecules oscillate up and down within the bounded areas defined by the two sinusoidal curves while propagating along the pipe. As time progresses, the two curves grow narrower at first, then pull apart, and grow narrower again, followed by pulling apart once more. These indicate air pressure decreases and increases alternately, with the narrower regions associated with higher pressure and the wider regions associated with lower pressure.

E. None of the above. (Please state your answer here:

11. In an open-open pipe, when the third harmonic standing wave is formed, which best describes the motion of the air molecules?

A. At one moment, air molecules move along one of the two sinusoidal curves while oscillating parallel to the pipe. A half cycle later, air molecules move along the other sinusoidal curve while oscillating parallel to the pipe.

B. At one moment, air molecules move along one of the two sinusoidal curves while oscillating perpendicular to the pipe. A half cycle later, air molecules move along the other sinusoidal curve while oscillating perpendicular to the pipe. 
C. At one moment, air molecules compress around the left and the right pressure antinodes and reduce density and pressure around the middle pressure antinode. A half cycle later, air molecules compress around the middle pressure antinode and reduce density and pressure around the left and the right pressure antinodes.

D. During the entire cycle, air molecules oscillate up and down within the bounded areas defined by the two sinusoidal curves while propagating along the pipe. As time progresses, the two curves grow narrower, then pull part, grow narrower again, and pull part once more. Finally, they grow narrower once more and pull apart again. These indicate the air pressure increases and decreases alternately, with the narrower regions associated with higher pressure and the wider regions associated with lower pressure.

E. None of the above. (Please state your answer here:

12. In an open-closed pipe, when the first harmonic standing sound wave is formed, which best describes the motion of the air molecules?

A. At one moment, air molecules move along one of the two sinusoidal curves while oscillating parallel to the pipe. A half cycle later, air molecules move along the other sinusoidal curve while oscillating parallel to the pipe.

B. At one moment, air molecules move along one of the two sinusoidal curves while oscillating perpendicular to the pipe. A half cycle later, air molecules move along the other sinusoidal curve while oscillating perpendicular to the pipe.

C. At one moment, air molecules compress towards the closed end of the pipe. A half cycle later, air molecules reduce density and pressure around the closed end of the pipe and extend themselves towards the open end of the pipe.

D. During the entire cycle, air molecules oscillate up and down within the bounded areas by the two sinusoidal curves while propagating along the pipe. As time progresses, the two curves grow narrower, indicating air pressure increases accordingly.

E. None of the above. (Please state your answer here:
13. In an open-closed pipe, when the third harmonic standing wave is formed, which best describes the motion of the air molecules?

A. At one moment, air molecules move along one of the two sinusoidal curves while oscillating parallel to the pipe. A half cycle later, air molecules move along the other sinusoidal curve while oscillating parallel to the pipe.

B. At one moment, air molecules move along one of the two sinusoidal curves while oscillating perpendicular to the pipe. A half cycle later, air molecules move along the other sinusoidal curve while oscillating perpendicular to the pipe.

C. At one moment, air molecules compress towards the closed end of the pipe and reduce density and pressure around the other pressure antinode. A half cycle later, air molecules compress around the other pressure antinode and reduce density and pressure near the open end and the closed end of the pipe.

D. During the entire cycle, air molecules oscillate up and down within the bounded areas by the two sinusoidal curves while propagating along the pipe. As time progresses, the curves grow narrower, then pull part, and grow narrower again, indicating the air pressure increases and decreases alternately, with the narrower regions associated with higher pressure and the wider regions associated with lower pressure.

E. None of the above. (Please state your answer here:

14. In an open-closed pipe, when the fifth harmonic standing wave is formed, which best describes the air molecule motions? 
A. At one moment, air molecules move along one of the two sinusoidal curves while oscillating parallel to the pipe. A half cycle later, air molecules move along the other sinusoidal curve while oscillating parallel to the pipe.

B. At one moment, air molecules move along one of the two sinusoidal curves while oscillating perpendicular to the pipe. A half cycle later, air molecules move along the other sinusoidal curve while oscillating perpendicular to the pipe.

C. At one moment, air molecules compress around the closed end of the pipe and the pressure antinode near the opening of the pipe and reduce density and pressure around the pressure antinode in between. A half cycle later, air molecules compress around the pressure antinode in between and reduce density and pressure around the pressure antinodes located at the closed end and near the opening of the pipe.

D. During the entire cycle, air molecules oscillate up and down within the bounded areas by the two sinusoidal curves while propagating along the pipe. As time progresses, the two curves grow narrower, pull apart, grow narrower again, and then pull apart once more. Finally, they grow narrower. These indicate that the air pressure increases and decreases alternately, with the narrower regions associated with higher pressure and the wider regions associated with lower pressure.

E. None of the above. (Please state your answer here:

\section{APPENDIX B: TEXTBOOK HANDOUT}

See Fig. 8.

\section{APPENDIX C: ANOTHER PERSPECTIVE}

(The authors created this handout from the general ideas contained in introductory physics textbooks [8-12] and from their self-designed illustrations with supporting text.)

Sound Standing Waves in an Air Column inside a Pipe

Sound is a longitudinal pressure wave which forms compression and rarefaction regions in a medium such as air. When sound waves propagate in a pipe, the air molecules move along the pipe and the ends of the pipe induce boundary conditions for the motion of the air

\subsection{STANDING WAVES IN AIR COLUMNS}

Standing longitudinal waves can be set up in a tube of air, such as an organ pipe, as the result of interference between sound waves traveling in opposite directions. The relationship between the incident wave and the reflected wave depends on whether the reflecting end of the tube is open or closed. A portion of the sound wave is re flected back into the tube even at an open end. If one end is closed, a node must exist at that end because the movement of air is restricted. If the end is open, the ele ments of air have complete freedom of motion, and an antinode exists.

Figure 14.23a shows the first three modes of vibration of a pipe open at both ends. When air is directed against an edge at the left, longitudinal standing wave are formed and the pipe vibrates at its natural frequencies. Note that, from end to end, the pattern is $\mathrm{A}-\mathrm{N}-\mathrm{A}$, the same pattern as in the vibrating string, except node and antinode have exchanged positions. As before, an antinode and its adjacent node, $\mathrm{A}-\mathrm{N}$, represent a quarter-wavelength, and there are two, $\mathrm{A}-\mathrm{N}$ and $\mathrm{N}-\mathrm{A}$, so $L=2\left(\lambda_{1} / 4\right)=\lambda_{1} / 2$ and $\lambda_{1}=2 L$. The fundamental frequency of the pipe open at both ends is then $f_{1}=v / \lambda_{1}=v / 2 L$. The next harmonic has an addi-

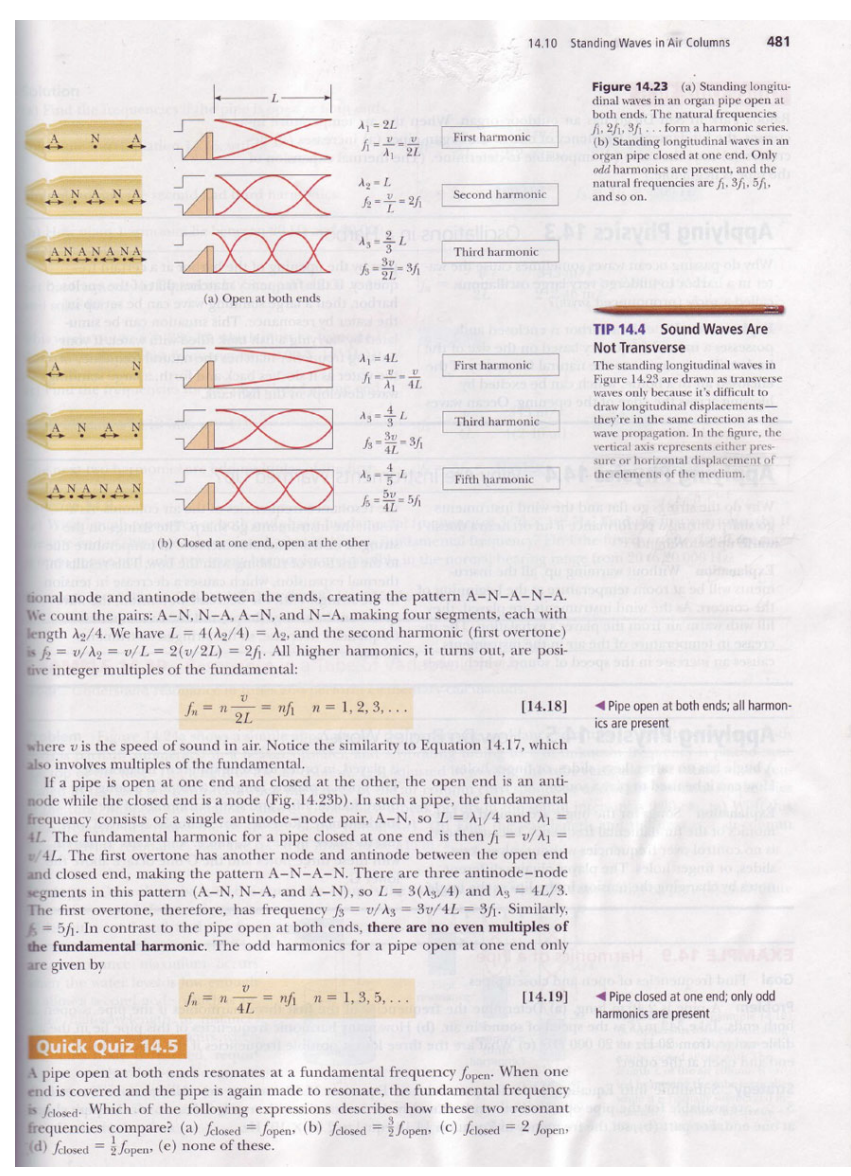

FIG. 8. Excerpt from a textbook [11] that contains diagrams for sound standing waves represented by two intertwining sinusoidal curves. This excerpt is reproduced by permission: From SERWAY/FAUGHN/VUILLE. Enhanced College Physics (with PhysicsNOW), 7E. (C) 2006 Brooks/Cole, a part of Cengage Learning, Inc. Reproduced by permission. www.cengage.com/ permissions.

molecules. At an open end, air pressure should be always equal to atmospheric pressure. Therefore, some air molecules are reflected back into the pipe while some air molecules are refracted out into the atmosphere. The reflected sound waves and the incident sound waves superpose on each other, which produce constructive and destructive interferences based on the principle of 
superposition. At a closed end, sound waves are fully reflected back off the closed end and superposed on the incident waves to form constructive and destructive interferences. Specifically, when the frequency of the sound waves and the length of the pipe are appropriately chosen, sound standing waves can be formed; pressure nodes represent places where there are no pressure deviations from atmospheric pressure, and pressure antinodes refer to the regions where pressure deviations from the atmosphere pressure vary between a maximum and a minimum. Sound standing waves are an example of standing longitudinal waves.

A sound standing wave of the most fundamental frequency in an open-open pipe, the first harmonic, is depicted in Fig. 9. There is one pressure antinode, which is located in the middle of the pipe. At one moment in time, air molecules squeeze together towards the middle of the pipe, which results in maximum pressure in the middle of the pipe, as depicted in Fig. 9(a). After that, they pull apart and extend towards both ends of the pipe, which results in air pressure and density decreasing in the middle of the pipe and, consequently, increasing in other regions of the pipe. Figure 9(b) shows that one-quarter cycle later the air pressure inside the tube is uniformly distributed for one moment. As air molecules further extend towards both ends of the pipe, the air pressure and density in the middle of the pipe eventually reach a minimum another quarter cycle later as shown in Fig. 9(c). From this state, air molecules start to squeeze towards the middle of the pipe again, reaching a maximum pressure and density in the middle of the pipe and repeating the process described above. Because the middle of the pipe experiences the maximum variation of pressure during one cycle, it is an antinode of the pressure. On the other hand, the air pressure at both open ends is always equal to atmospheric pressure, and thus they are pressure nodes.
Equivalently, sound standing waves can be analyzed in terms of displacement variations of air molecules as a function of space and time. The displacement curves in Figs. 9(a) through 9(c) show how air molecules in any arbitrary point along the pipe deviate from the equilibrium position (the initial $x$ coordinate of the point). Positive displacement (along the $x$-axis direction) refers to air molecules displacing to the right, and negative displacement refers to air molecules displacing to the left. For example, the displacement curve in Fig. 9(a) depicts that air molecules in the left half of the pipe move to the right towards the middle of the pipe, while the air molecules in the right half of the pipe move to the left towards the middle of the pipe. This results in the increase of air pressure in the middle of the pipe. Comparing the pressure curves with the corresponding displacement curves in Fig. 9, one can see that a pressure antinode is a displacement node, and a pressure node is a displacement antinode. As time elapses, the amplitudes of the pressure and displacement curves gradually vary between the shapes depicted in Fig. 9(a) and the ones in Fig. 9(c). Since the wavelength of the displacement curve or the pressure curve is twice the length of the pipe, the fundamental frequency of the sound standing wave in the open-open pipe is $f_{1}=\frac{v}{\lambda}=\frac{v}{2 L}$.

The sound standing waves of the next higher frequency, the second harmonic, have two pressure antinodes as shown in Fig. 10. This, or higher harmonic standing waves, can be analyzed in the same fashion as the first harmonic in terms of pressure and displacement. At one moment, air molecules squeeze towards a pressure antinode on the left and reduce density and pressure around the pressure antinode on the right, as indicated in Fig. 10(a). A half cycle later, air molecules reduce density and pressure around the pressure antinode on the left and compress around the pressure antinode on the right as shown in Fig. 10(c). Therefore, air molecules move back and forth in
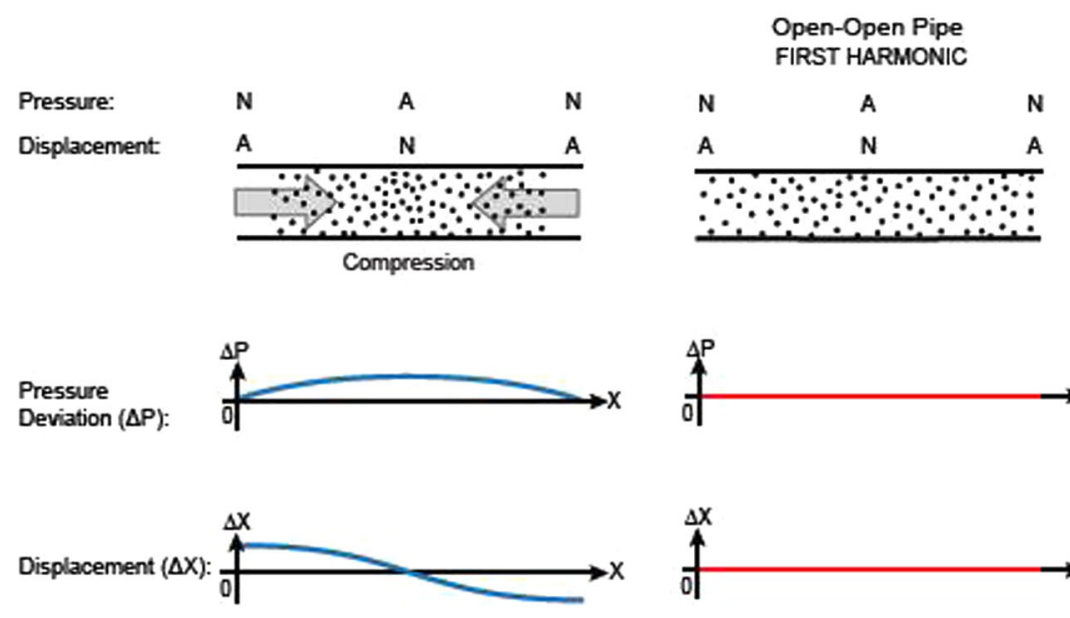

(a) At one moment

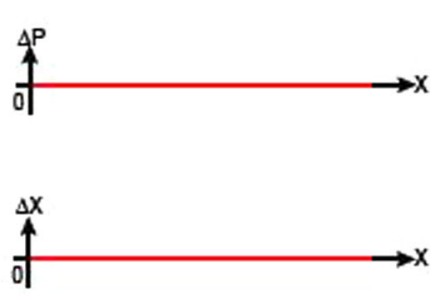

(b) A quarter cycle later than (a)
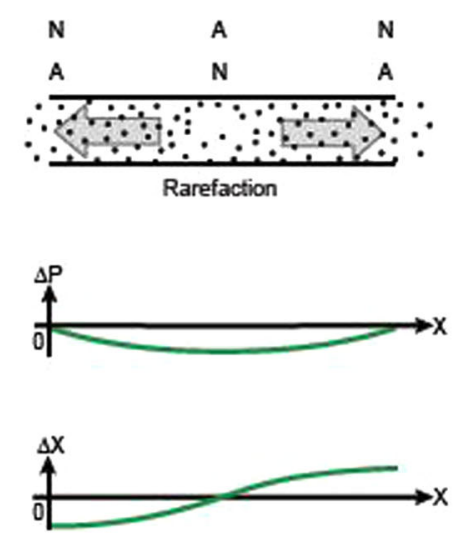

(c) A half cycle later than (a)

FIG. 9. First harmonic standing sound wave in an open-open pipe. 


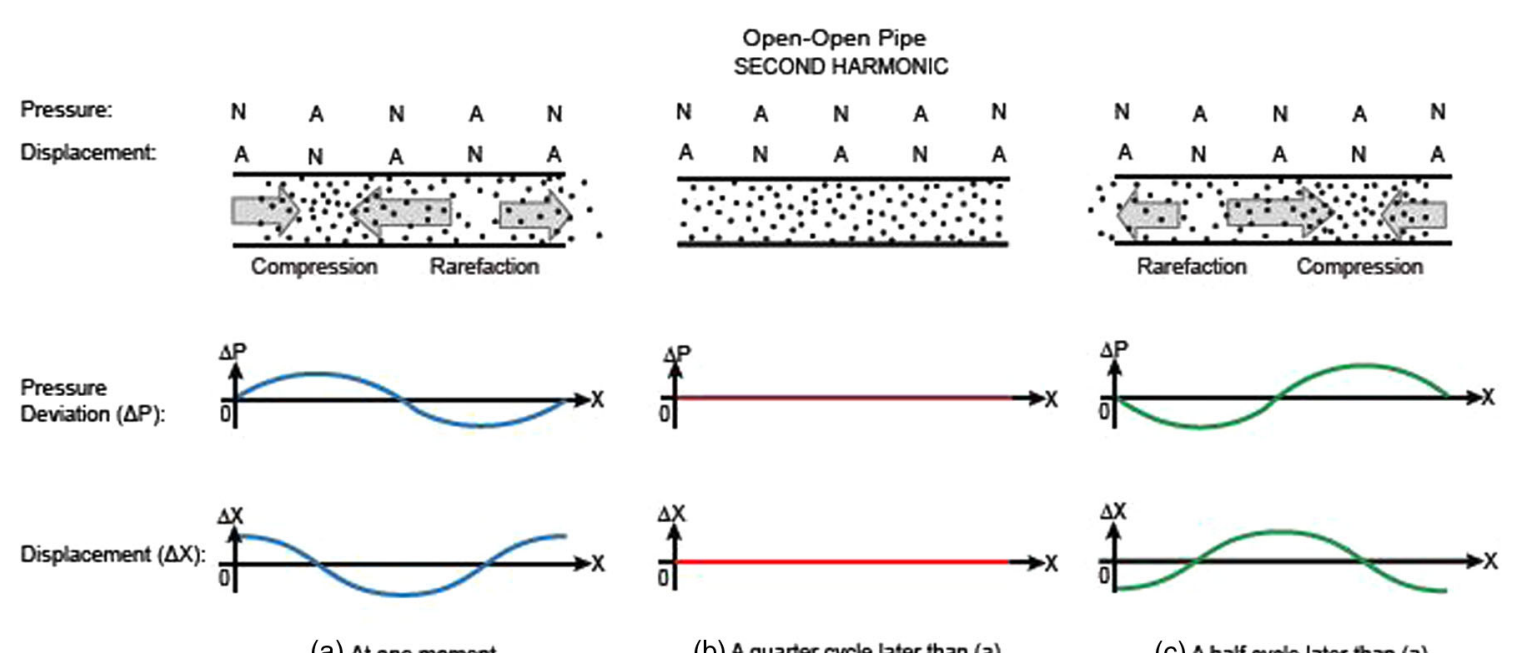

(a) At one moment

(b) A quarter cycle later than (a)

(c) A half cycle later than (a)

FIG. 10. Second harmonic sound standing wave in an open-open pipe.

three sections of the pipe separated by the two pressure antinodes. Since the wavelength of the displacement curve or the pressure curve equals the length of the pipe, the corresponding frequency $f_{2}$ is twice the fundamental frequency, $f_{2}=v / L=2 f_{1}$.

The sound standing waves of an even higher frequency, the third harmonic, have three pressure antinodes. At one moment, air molecules compress around the two pressure antinodes on the left and the right of the pipe at the same time, while reducing density and pressure around the middle pressure antinode as shown in Fig. 11(a). A half cycle later, air molecules compress around the middle pressure antinode and reduce density and pressure around the two pressure antinodes on the left and the right, as illustrated in Fig. 11(c). Since the wavelength of the displacement curve or the pressure curve equals $\frac{2}{3}$ of the length of the pipe, the corresponding frequency of the sound standing wave $f_{3}$ is three times the fundamental frequency, $f_{3}=v / \lambda=v / \frac{2}{3} L=3 f_{1}$. Therefore, it can be inferred that all higher harmonics are positive integer multiples of the first harmonic:

$$
f_{n}=n \frac{v}{2 L}=n f_{1} \quad(n=1,2,3, \ldots) .
$$

In the open-closed pipe, the closed end is always a pressure antinode because the air molecules compress there due to it being a hard boundary. On the standing waves of the fundamental frequency, the first harmonic, air molecules at one moment squeeze towards the left closed end, as seen in Fig. 12(a). A half cycle later, air molecules reduce density and pressure near the closed end and extend towards the open end, passing through the stage depicted by Fig. 12(b) and eventually reaching the state depicted in Fig. 12(c). Since the wavelength of the displacement curve or the pressure curve is four times the length of the pipe, the fundamental frequency of the standing sound wave in the open-closed pipe is $f_{1}^{\prime}=v / \lambda=v / 4 L$.

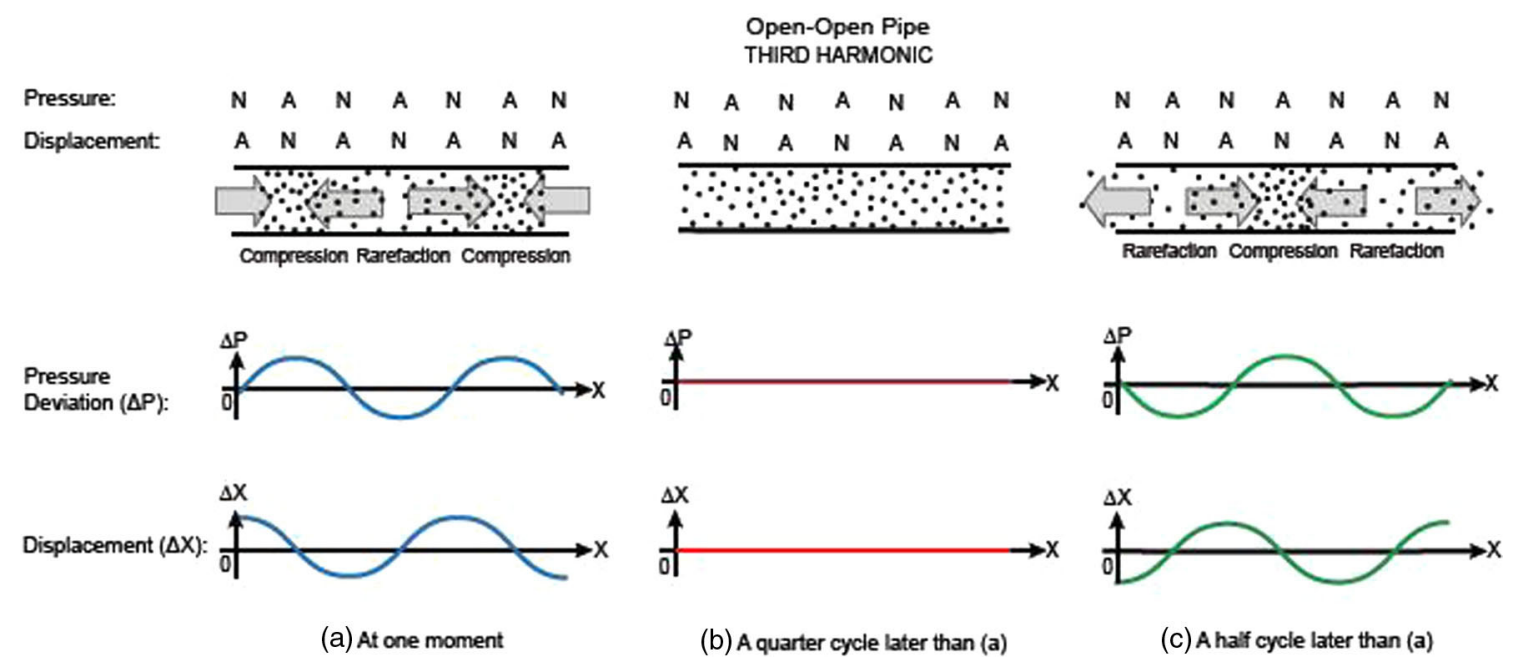

FIG. 11. Third harmonic sound standing wave in an open-open pipe. 


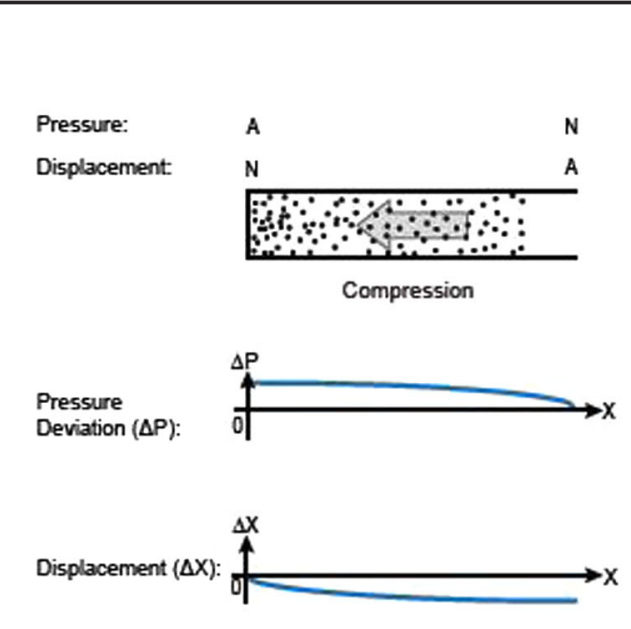

(a) At one moment
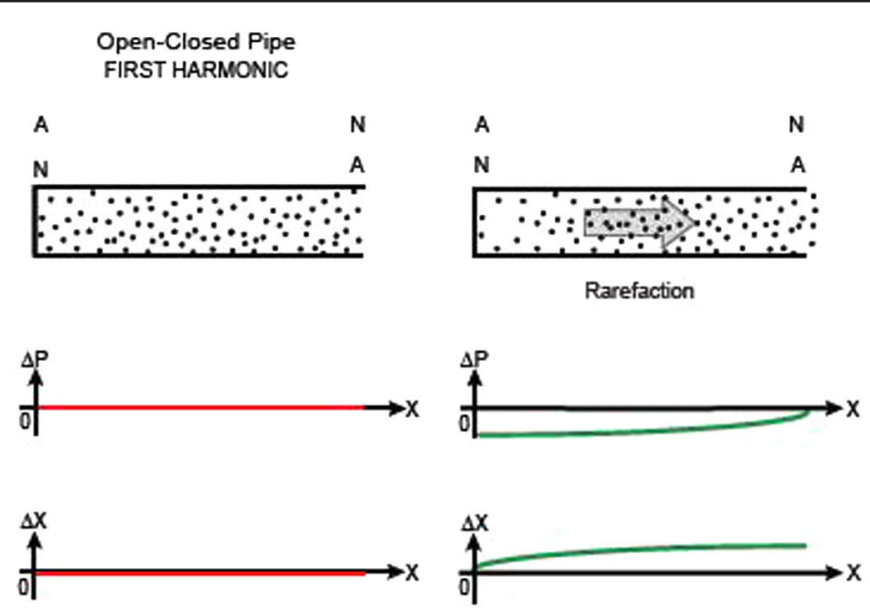

(b) A quarter cycle later than (a)

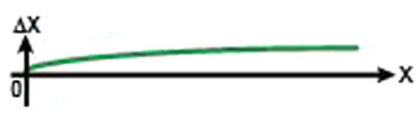

(c) A half cycle later than (a)

FIG. 12. First harmonic sound standing wave in an open-closed pipe.

For the standing wave of the next higher frequency, the third harmonic, there are two pressure antinodes, which include the one at the closed end. At one moment in time, the air molecules squeeze towards the left closed end of the tube and reduce density and pressure around the pressure antinode on the right, as shown in Fig. 13(a). A half cycle later, air molecules squeeze towards the pressure antinode on the right and reduce density and pressure near the closed end, as illustrated in Fig. 13(c). Since the wavelength of the displacement curve or the pressure curve equals $\frac{4}{3}$ of the length of the pipe, the corresponding frequency of the sound standing wave $f_{3}^{\prime}$ is three times the fundamental frequency $f_{3}^{\prime}=v / \lambda=v / \frac{4}{3} L=3 v / 4 L=3 f_{1}^{\prime}$. This is why it is called the third harmonic.

For the standing wave of the next higher frequency, the fifth harmonic, there are three pressure antinodes including the one at the closed end. At one moment, air molecules compress towards the left closed end, reduce density and pressure around the middle pressure antinode, and squeeze towards the pressure antinode on the right, as shown in Fig. 14(a). A half cycle later, the air molecules reduce density and pressure near the left closed end of the pipe, compress around the middle pressure antinode, and reduce density and pressure around the pressure antinode on the right, as shown in Fig. 14(c). Since the wavelength of the displacement curve or the pressure curve equals $\frac{4}{5}$ of the length of the pipe, the corresponding frequency of the sound standing wave is five times the fundamental frequency $f_{5}^{\prime}=v / \lambda=v / \frac{4}{5} L=5 v / 4 L=5 f_{1}^{\prime}$. This is why it is called the fifth harmonic. It can be inferred that the odd harmonics for an open-closed pipe are given by

$$
f_{n}^{\prime}=n v / 4 L=n f_{1}^{\prime} \quad(n=1,3,5, \ldots) .
$$

As sound is a pressure wave, the amplitude of the displacement or pressure curves is only related to the

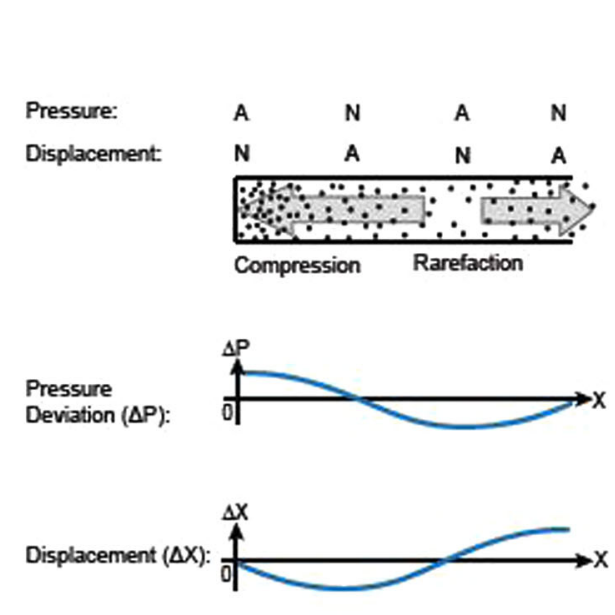

(a) At one moment

\begin{abstract}
Open-Closed Pipe
THIRD HARMONIC
\end{abstract}

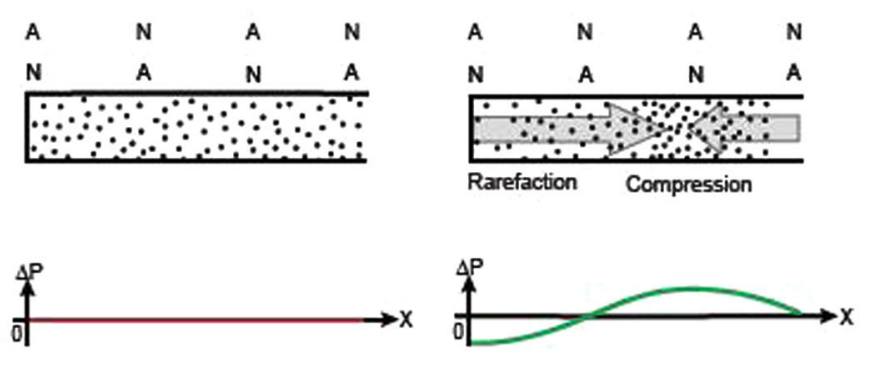

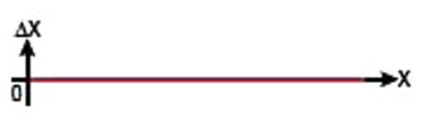

(b) A quarter cycle later than (a)

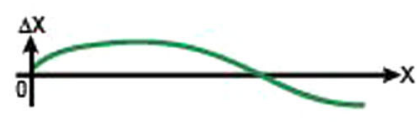

(c) A half cycle later than (a)

FIG. 13. Third harmonic sound standing wave in an open-closed pipe. 


\section{Open-Closed Pipe
FIFTH HARMONIC}

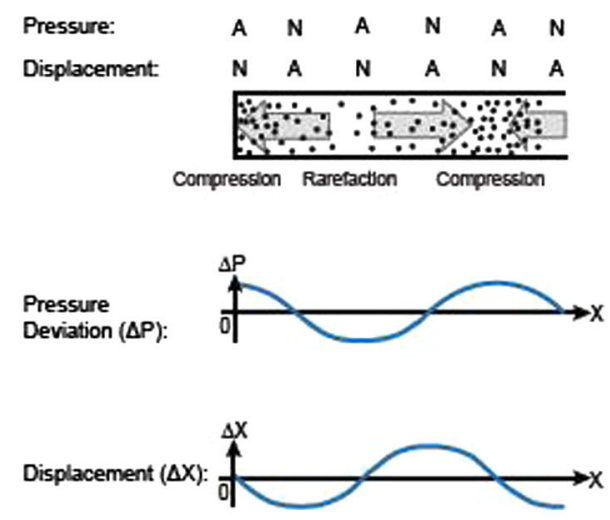

(a) At one moment
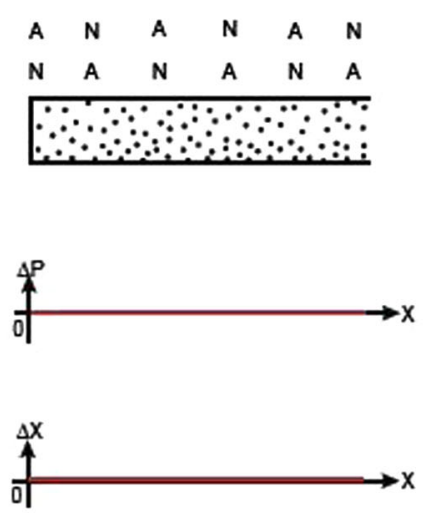

(b) A quarter cycle later than (a)
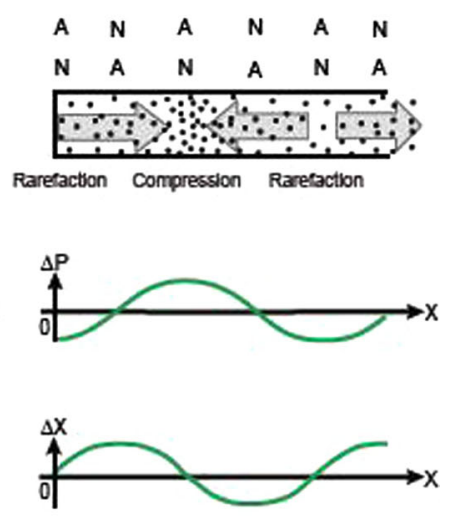

(c) A half cycle later than (a)

FIG. 14. Fifth harmonic sound standing wave in an open-closed pipe.

magnitude of the pressure produced by the sound source and is not related to the diameter of the pipe.

\section{APPENDIX D: POST ALT SURVEY}

Instruction: After bubbling in your answer for each question on your Scantron, please provide your reason, basis, or source for your answer to each question on the blank line. Sources may include, for example, specific page(s) in the text of the handout, specific figure numbers in the handout, or your previous knowledge.

1. Which of the following best describes a sound standing wave?

A. It is a longitudinal wave.

B. It is a transverse wave.

C. Sometimes transverse sometimes longitudinal.

D. It is an electromagnetic wave.

2. Which of the following is TRUE about the motion of the air molecules when a sound standing wave is formed in an air column inside a pipe?

I. The air molecules oscillate perpendicular to the pipe.

II. The air molecules oscillate parallel to the pipe.

III. The air molecules move along the pipe in a sinusoidal curve.

A. I only, B. II only, C. III only, D. I \& III, E. II \& III.
3. Refer to Figs. 9-14 in the handout only. Which of the following is depicted by the six figures about the diameter of the pipe and the amplitude of the sound standing waves?

A. The amplitude of the sound standing waves is greater than the diameter of the pipe.

$B$. The amplitude of the sound standing waves is smaller than the diameter of the pipe.

C. The amplitude of the sound standing waves is equal to the diameter of the pipe.

D. The amplitude of the sound standing waves is not at all related to the diameter of the pipe.

4. How does the amplitude of the sound standing waves relate to the diameter of the pipe in your opinion?

A. The amplitude of the sound standing waves is greater than the diameter of the pipe.

B. The amplitude of the sound standing waves is smaller than the diameter of the pipe.

C. The amplitude of the sound standing waves is equal to the diameter of the pipe.

D. The amplitude of the sound standing waves is not at all related to the diameter of the pipe.

5. Which of the following is TRUE about the displacement antinode in a sound standing wave in an air column inside a pipe? 
A. The displacement antinode depicts where the displacement of air molecules can have the largest variation from the equilibrium position in a direction parallel to the pipe.

B. The displacement antinode depicts where the displacement of air molecules from the equilibrium position is always at a maximum in a direction parallel to the pipe.

C. The displacement antinode depicts where the displacement of air molecules can have the largest variation from the equilibrium position in a direction perpendicular to the pipe.

D. The displacement antinode depicts where the displacement of air molecules from the equilibrium position is always at a maximum in a direction perpendicular to the pipe.

6. Which of the following is TRUE about the pressure node and pressure antinode in a sound standing wave in an air column inside a pipe?

I. The pressure node in a sound standing wave is where the air pressure is zero.

II. The pressure node in a sound standing wave is where the air pressure has no deviation from atmospheric pressure.

III. The pressure antinode in a sound standing wave is where the air pressure is always at a maximum.

IV. The pressure antinode in a sound standing wave is where the air pressure has the largest variation between a maximum compression (air pushing together) and a maximum rarefaction (air pulling apart).

A. I and III, B. I and IV, C. II and III, D. II and IV.

7. Which of the following is TRUE about a sound standing wave in an air column inside a pipe with two open ends?

I. The open ends of the air column are pressure nodes.

II. The open ends of the air column are pressure antinodes.

III. The open ends of the air column are displacement nodes.

IV. The open ends of the air column are displacement antinodes.

A. I and III, B. I and IV, C. II and III, D. II and IV.
8. Which of the following is TRUE about a sound standing wave in an air column inside a pipe with one closed end and one open end?

I. The open end of the air column is a pressure node.

II. The open end of the air column is a pressure antinode.

III. The open end of the air column is a displacement node.

IV. The open end of the air column is a displacement antinode.

A. I and III, B. I and IV, C. II and III, D. II and IV.

9. Which of the following is TRUE about a sound standing wave in an air column inside a pipe with one closed end and one open end?

I. The closed end of the air column is a pressure node.

II. The closed end of the air column is a pressure antinode.

III. The closed end of the air column is a displacement node.

IV. The closed end of the air column is a displacement antinode.

A. I and III, B. I and IV, C. II and III, D. II and IV.

10. In an open-open pipe, when the first harmonic sound standing wave is formed, which best describes the motion of the air molecules?

A. At one moment, air molecules move along one of the two sinusoidal curves while oscillating parallel to the pipe. A half cycle later, air molecules move along the other sinusoidal curve while oscillating parallel to the pipe.

B. At one moment, air molecules move along one of the two sinusoidal curves while oscillating perpendicular to the pipe. A half cycle later, air molecules move along the other sinusoidal curve while oscillating perpendicular to the pipe.

C. At one moment, air molecules compress towards the center of the pipe. A half cycle later, air molecules reduce pressure and density around the center of the pipe and extend themselves towards both ends. 
D. During the entire cycle, air molecules oscillate up and down within the bounded areas defined by the two sinusoidal curves while propagating along the pipe. As time progresses, the two curves grow narrower around the center and then pull apart, indicating air pressure increases and then decreases accordingly, with the narrower regions associated with higher pressure and the wider regions associated with lower pressure.

E. None of the above. (Please state your answer here:

11. In an open-open pipe, when the second harmonic standing wave is formed, which best describes the motion of the air molecules?

A. At one moment, air molecules move along one of the two sinusoidal curves while oscillating parallel to the pipe. A half cycle later, air molecules move along the other sinusoidal curve while oscillating parallel to the pipe.

B. At one moment, air molecules move along one of the two sinusoidal curves while oscillating perpendicular to the pipe. A half cycle later, air molecules move along the other sinusoidal curve while oscillating perpendicular to the pipe.

C. At one moment, air molecules compress towards the left pressure antinode and reduce density and pressure around the right pressure antinode. A half cycle later, air molecules reduce density and pressure around the left pressure antinode and compress around the right pressure antinode.

D. During the entire cycle, air molecules oscillate up and down within the bounded areas defined by the two sinusoidal curves while propagating along the pipe. As time progresses, the two curves grow narrower at first, then pull apart, and grow narrower again, followed by pulling apart once more. These indicate air pressure decreases and increases alternately, with the narrower regions associated with higher pressure and the wider regions associated with lower pressure.

E. None of the above. (Please state your answer here:
12. In an open-open pipe, when the third harmonic standing wave is formed, which best describes the motion of the air molecules?

A. At one moment, air molecules move along one of the two sinusoidal curves while oscillating parallel to the pipe. A half cycle later, air molecules move along the other sinusoidal curve while oscillating parallel to the pipe.

B. At one moment, air molecules move along one of the two sinusoidal curves while oscillating perpendicular to the pipe. A half cycle later, air molecules move along the other sinusoidal curve while oscillating perpendicular to the pipe.

C. At one moment, air molecules compress around the left and the right pressure antinodes and reduce density and pressure around the middle pressure antinode. A half cycle later, air molecules compress around the middle pressure antinode and reduce density and pressure around the left and the right pressure antinodes.

D. During the entire cycle, air molecules oscillate up and down within the bounded areas defined by the two sinusoidal curves while propagating along the pipe. As time progresses, the two curves grow narrower, then pull part, grow narrower again, and pull part once more. Finally, they grow narrower once more and pull apart again. These indicate the air pressure increases and decreases alternately, with the narrower regions associated with higher pressure and the wider regions associated with lower pressure.

E. None of the above. (Please state your answer here:

13. In an open-closed pipe, when the first harmonic standing sound wave is formed, which best describes the motion of the air molecules? 
A. At one moment, air molecules move along one of the two sinusoidal curves while oscillating parallel to the pipe. A half cycle later, air molecules move along the other sinusoidal curve while oscillating parallel to the pipe.

B. At one moment, air molecules move along one of the two sinusoidal curves while oscillating perpendicular to the pipe. A half cycle later, air molecules move along the other sinusoidal curve while oscillating perpendicular to the pipe.

C. At one moment, air molecules compress towards the closed end of the pipe. A half cycle later, air molecules reduce density and pressure around the closed end of the pipe and extend themselves towards the open end of the pipe.

D. During the entire cycle, air molecules oscillate up and down within the bounded areas by the two sinusoidal curves while propagating along the pipe. As time progresses, the two curves grow narrower, indicating air pressure increases accordingly.

E. None of the above. (Please state your answer here:

14. In an open-closed pipe, when the third harmonic standing wave is formed, which best describes the motion of the air molecules?

A. At one moment, air molecules move along one of the two sinusoidal curves while oscillating parallel to the pipe. A half cycle later, air molecules move along the other sinusoidal curve while oscillating parallel to the pipe.

B. At one moment, air molecules move along one of the two sinusoidal curves while oscillating perpendicular to the pipe. A half cycle later, air molecules move along the other sinusoidal curve while oscillating perpendicular to the pipe.

C. At one moment, air molecules compress towards the closed end of the pipe and reduce density and pressure around the other pressure antinode. A half cycle later, air molecules compress around the other pressure antinode and reduce density and pressure near the open end and the closed end of the pipe.

D. During the entire cycle, air molecules oscillate up and down within the bounded areas by the two sinusoidal curves while propagating along the pipe. As time progresses, the curves grow narrower, then pull part, and grow narrower again, indicating the air pressure increases and decreases alternately, with the narrower regions associated with higher pressure and the wider regions associated with lower pressure.

E. None of the above. (Please state your answer here:

15. In an open-closed pipe, when the fifth harmonic standing wave is formed, which best describes the air molecule motions?

A. At one moment, air molecules move along one of the two sinusoidal curves while oscillating parallel to the pipe. A half cycle later, air molecules move along the other sinusoidal curve while oscillating parallel to the pipe.

B. At one moment, air molecules move along one of the two sinusoidal curves while oscillating perpendicular to the pipe. A half cycle later, air molecules move along the other sinusoidal curve while oscillating perpendicular to the pipe.

C. At one moment, air molecules compress around the closed end of the pipe and the pressure antinode near the opening of the pipe and reduce density and pressure around the pressure antinode in between. A half cycle later, air molecules compress around the pressure antinode in between and reduce density and pressure around the pressure antinodes located at the closed end and near the opening of the pipe.

D. During the entire cycle, air molecules oscillate up and down within the bounded areas by the two sinusoidal curves while propagating along the pipe. As time progresses, the two curves grow narrower, pull apart, grow narrower again, and then pull apart once more. Finally, they grow narrower. These indicate that the air pressure increases and decreases alternately, with the narrower regions associated with higher pressure and the wider regions associated with lower pressure.

E. None of the above. (Please state your answer here: 
the diameter of the pipe and the amplitude of the sound standing waves?

A. The amplitude of the sound standing waves is greater than the diameter of the pipe.

B. The amplitude of the sound standing waves is smaller than the diameter of the pipe.

C. The amplitude of the sound standing waves is equal to the diameter of the pipe.

D. The amplitude of the sound standing waves is

Note: Post Text survey includes 15 survey questions that not at all related to the diameter of the pipe

are identical to those in the Post Alt survey except that question 3 in the Post Text survey is replaced with the following question:

3. Refer to Fig. 14.23 on page 481 in the handout only. Which of the following is depicted by the six figures about

[1] R. J. Beichner, Testing student interpretation of kinematics graphs, Am. J. Phys. 62, 750 (1994).

[2] W. M. Roth and G. M. Bowen, Complexities of graphical representations during ecology lectures: An analysis rooted in semiotics and hermeneutic phenomenology, Learn. Instr. 9, 235 (1999).

[3] A. Elby, What students' learning of representations tells us about constructivism, J. Math. Behav. 19, 481 (2000).

[4] C. J. Linder, Understanding sound: So what is the problem?, Phys. Educ. 27, 258 (1992).

[5] N. S. Podolefsky and N. D. Finkelstein, Use of analogy in learning physics: The role of representations, Phys. Rev. ST Phys. Educ. Res. 2, 020101 (2006).

[6] M. C. Wittmann, R. N. Steinburg, and E. F. Redish, Understanding and affecting student reasoning about sound waves, Int. J. Sci. Educ. 25, 991 (2003).

[7] Z. Hrepic, D. A. Zollman, and N. J. Robello, Identifying students' mental models of sound propagation: The role of conceptual blending in understanding conceptual change, Phys. Rev. ST Phys. Educ. Res. 6, 020114 (2010).

[8] R. D. Knight, Physics for Scientists and Engineers (Pearson/Addison Wesley, San Francisco, CA, 2004), pp. 656-657.

[9] R. D. Knight, B. Jones, and S. Field, College Physics (Pearson/Addison Wesley, San Francisco, CA, 2004), pp. 524-525.

[10] D. C. Giancoli, Physics (Pearson/Prentice Hall, Upper Saddle River, NJ, 2005), pp. 332-333.

[11] R. A. Serway, J. S. Faughn, C. Vuille, and C. A. Bennett, College Physics (Thomson Brooks/Cole, Belmont, CA, 2006), pp. 480-481.

[12] A. Giambattista, B. M. Richardson, and R. C. Richardson, College Physics (McGraw-Hill, New York, NY, 2007), pp. 424-426.

[13] M. Alonso and E. J. Finn, Physics (Addison Wesley, Longman Limited, Edinburg Gate, England, 1992).
[14] A. Beiser, Physics (Addison Wesley, Reading, MA, 1992).

[15] E. R. Huggins, Physics 2000 (Moose Mountain Digital Press, Etna, New Hampshire, 1999).

[16] J. D. Wilson, A. J. Buffa, and B. Lou, College Physics (Pearson Addison-Wesley, San Francisco, CA, 2001).

[17] J.S. Walker, Physics (Pearson Education, Inc., Upper Saddle River, New Jersey, 2004).

[18] H. D. Young and G. R. Geller, College Physics (Addison Wesley, San Francisco, CA, 2006).

[19] R. A. Serway and J. W. Jewett, Physics for Scientists and Engineers (Thomson Higher Education, Belmont, CA, 2008).

[20] J. D. Cutnell and K. W. Johnson, Physics (John Wiley \& Sons, Inc., Hoboken, NJ, 2009).

[21] D. A. Griffith, Physics Labs with Computers, Teacher's Guide Vol. 2 (PASCO Scientific, Roseville, CA, 1999), p. 97.

[22] L. Zeng, E. Corpuz, and R. Rosalez, Pictorial representations of longitudinal standing waves in AAPT Summer Meeting Program 2009, Ann Arbor, Michigan (American Association of Physics Teachers, College Park, MD, 2009).

[23] L. Zeng, E. Corpuz, R. Rosalez, J. Rodriguez, and I. Abundiz, Illustrations of sound standing waves in air columns in introductory physics textbooks in $A A P T$ Summer Meeting Program 2010, Portland, Oregon (American Association of Physics Teachers, College Park, MD, 2010).

[24] D. P. Ausubel, A cognitive structure view of word and concept meaning, in Readings in the Psychology of Cognition edited by R.C. Anderson and D.P. Ausubel (Holt, Rinehart and Winston, New York, 1965), pp. $58-75$.

[25] D. P. Ausubel, In defense of verbal learning, Readings in the Psychology of Cognition, edited by R. C. Anderson and D. P. Ausubel (Holt, Rinehart and Winston, New York, 1965), pp. 87-102. 
[26] D. P. Ausubel, Cognitive structure and the facilitation of meaningful verbal learning, in Readings in the Psychology of Cognition, edited by R. C. Anderson and D. P. Ausubel (Holt, Rinehart and Winston, New York, 1965), pp. $103-115$.

[27] D. P. Ausubel, The facilitation of meaningful verbal learning in the classroom, Educ. Psychol. 12, 162 (1977).

[28] R. E. Mayer, Rote versus meaningful learning, Theory Into Practice 41, 226 (2002).

[29] J. D. Novak, Practice in application of learning theory, Theory Into Practice 19, 58 (1980).

[30] J. D. Novak, Meaningful learning: The essential factor for conceptual change in limited or inappropriate propositional hierarchies leading to empowerment of learners, Sci. Educ. 86, 548 (2002).

[31] G. Wong, Meaningful GP learning with concept mapping, Educ. Prim. Care., 23, 300 (2012).

[32] H. Mandl and J. R. Levin, Knowledge acquisition from Text and Pictures (Elsevier Science Publishers, B.V., Amsterdam, 1989).

[33] R. Mayer, Systematic thinking fostered by illustrations in scientific text, J. Educ. Psychol. 81, 240 (1989).

[34] G. Deledalle, Charles S. Peirce's Philosophy of Signs (Indiana University Press, Bloomington, IN, 2000).

[35] K. J. Jamani, A semiotics discourse analysis framework: Understanding meaning making in science, in Semiotics Theory and Applications, edited by S. C. Hamel (Nova Science Publishers, Inc., New York, NY, 2011), pp. 191-208.

[36] S. C. Hamel, Semiotics: Theory and Applications (Nova Science Publishers, Inc., New York, 2011).

[37] D. Chandler, Semiotics: The Basics (Routledge, New York, 2002).

[38] M. D. Gall, J.P. Gall, and W. R. Borg, Educational Research: An Introduction (Pearson Education, Inc., Boston, MA, 2003).
[39] R. Rosenthal and R. L. Rosnow, The Volunteer Subject (John Wiley \& Sons, Inc., New York, 1975).

[40] L. Zeng, E. Corpuz, C. Smith, and J. Rodriguez , New pictorial representations and supporting text of sound standing waves of air columns in a tube, in AAPT Summer Meeting Program 2011, Omaha, Nebraska (American Association of Physics Teachers, College Park, MD, 2011).

[41] W.W. Seto, Schaum's Outline Series: Theory and Problems of Acoustics (McGraw-Hill, New York, 1971), pp. 53-56.

[42] A. B. Wood, A Textbook of Sound (G. Bell and Sons, Ltd., London, Great Britain, 1960), pp. 179-182.

[43] A. Wood, Acoustics (Dover Publications, Inc., New York, 1966), pp. 55-56.

[44] M. P. Morse, Vibration and Sound (McGraw-Hill Company, Inc., New York, NY, 1948), pp. 219-222.

[45] D. T. Rossing, Handbook of Acoustics (Springer: Science +Business Media, LLC, New York, 2007), p. 602.

[46] W. Winn, The Design and use of instructional graphics, in Knowledge Acquisition from Text and Pictures, edited by H. Mandl and J. R. Levin (Elsevier Science Publishers, B.V., Amsterdam, 1989), pp. 127-131.

[47] R. E. Mayer and J. K. Gallini, When is an illustration worth ten thousand words?, J. Educ. Psychol. 82, 715 (1990).

[48] S. H. Broughton, G. M. Sinatra, and R. E. Reynolds, The nature of the refutation text effect: An investigation of attention allocation, J. Educ. Res. 103, 407 (2010).

[49] J. R. Fraenkel and N.E. Wallen, How to Design and Evaluate Research in Education (McGraw-Hill Company, Inc., New York, NY, 2003), p. 279.

[50] N. A. Weiss, Introductory Statistics (Pearson Education, Inc., Boston, MA, 2012), pp. 566-568.

[51] N. A. Weiss, Introductory Statistics (Pearson Education, Inc., Boston, MA, 2012), pp. 557-561. 